\title{
Iwahori's Question for Affine Hecke Algebras
}

\author{
Toshiaki Shoji* and Nanhua $\mathrm{Xi}^{\dagger}$
}

\begin{abstract}
In this paper we show that an affine Hecke algebra $H_{q}$ over complex numbers field with parameter $q \neq 1$ is not isomorphic to the group algebra over complex numbers field of the corresponding extend affine Weyl group if the corresponding root system has no factors of type $A_{1}$ and the order of $q$ is different from 11 and 13 if the root system has factors of type $E_{8}$.
\end{abstract}

For a Hecke algebra (over a field) of a finite Coxeter group, it is known that the Hecke algebra is isomorphic to the group algebra over the field of complex numbers when both the Hecke algebra and the group algebra are semisimple. For a simple criterion of the semisimplicity for the Hecke algebra we refer to [G]. It is natural to consider the question for affine Hecke algebra, this question is analogue to a question of Iwahori for Weyl group[I]. For affine Hecke algebras the answer is different. In an unpublished work around 1970's, Casselman proved, by using group cohomology, that the Hecke algebra of a simple group over a $p$-adic field $k$ with respect to an Iwahori subgroup is not isomorphic to the group algebra of the corresponding affine Weyl group. unless the group is of $k$-rank one. (The authors thank the referee for pointing out this fact.) We will show that an affine Hecke algebra $H_{q}$ over the complex number field with parameter $q \neq 1$ is not isomorphic to the group algebra over complex numbers field of the corresponding extend affine Weyl group if the the root system has no factors of type $A_{1}$. For technical reason, we require that the order of $q$ is different from 11 and 13 if the root system has factors of type $E_{8}$, see Theorem 1.2 . 


\section{Affine Hecke algebras}

1.1. Let $R$ be an irreducible root system, $W_{0}$ the Weyl group of $R$, $Q=\mathbb{Z} R$ the root lattice and $X$ the weight lattice. The Weyl group $W_{0}$ acts on $Q$ and $X$. Then the semidirect product $W_{a}=W_{0} \ltimes Q$ is an affine Weyl group, which is a subgroup of the extend affine Weyl group $W=W_{0} \ltimes X$. Fix a positive root system of $R$ and denote by $R^{+}$the set of positive roots of $R$. Then we have a length function $l: W \rightarrow \mathbb{N}$ given by the formula (see [IM])

$$
l(w x)=\sum_{\substack{\alpha \in R^{+} \\ w(\alpha) \in R^{-}}}\left|\left\langle x, \alpha^{\vee}\right\rangle+1\right|+\sum_{\substack{\alpha \in R^{+} \\ w(\alpha) \in R^{+}}}\left|\left\langle x, \alpha^{\vee}\right\rangle\right|,
$$

where $R^{-}=-R^{+}$. The set $S$ of simple reflections consists of all elements in $W_{a}$ with length 1 . The set of dominant weights $X^{+}$is $\{x \in X \mid l(w x)=l(w)+l(x)\}$.

Denote by $H_{q}$ the Hecke algebra of $(W, S)$ over the complex numbers field $\mathbb{C}$ with a non zero parameter $q \in \mathbb{C}^{*}$. By definition, $H_{q}$ has a $\mathbb{C}$ basis consisting of elements $T_{w}, w \in W$, and the multiplication law is given by the relations: $\left(T_{r}-q\right)\left(T_{r}+1\right)=0$ if $r \in S, T_{w} T_{u}=T_{w u}$ if $l(w u)=l(w)+l(u)$.

The main result of this article is the following.

Theorem 1.2. Let $q \neq 1$ be a nonzero complex number and $R$ an irreducible root system of rank greater than 1 . Assume that the order of $q$ is different from 11 and 13 if $R$ is of type $E_{8}$. Then $H_{q}$ is not isomorphic to $\mathbb{C}[W]$.

Remark. When $W$ is of type $\tilde{A}_{2}$, the result was proved in $[\mathrm{X} 1$, 11.7]. When $W$ is of type $\tilde{A}_{1}$, we know that $H_{q}$ is isomorphic to $\mathbb{C}[W]$ if and only if $q \neq-1$, see loc.cit.. For type $\tilde{A}_{2}$, Yan showed that $H_{q}$ is isomorphic to $H_{p}$ if and only if $p=q$ or $p q=1$.

The theorem should be valid for $R$ being of type $E_{8}$ and the order of $q$ is 11 or 13 , but the authors have not been able to work out it. Recently the authors learned that this result is known to G. Lusztig for long time. 
1.3. The center of $H_{q}$ will play a key role in the proof. We need to recall the description of Bernstein for the center.

For each $x$ in $X$, we can find $y$ and $z$ in $X^{+}$such that $x=y z^{-1}$. Define $\theta_{x}=q^{\frac{1}{2}(l(z)-l(y))} T_{y} T_{z}^{-1}$. It is known that $\theta_{x}$ is independent of the choice of $y$ and $z$.

(a) (Bernstein) For any $x, y$ in $X, \theta_{x} \theta_{y}=\theta_{y} \theta_{x}=\theta_{x y}$. The elements $\theta_{x}, x \in X$ form a $\mathbb{C}$-basis of the subalgebra $\Theta_{q}$ of $H_{q}$ generated by all $\theta_{y}, y \in X$. The Hecke algebra $H_{q}$ is a free $\Theta_{q}$-module with a basis $T_{w}, w \in W_{0}$.

For each dominant weight $x$ in $X$, let $O_{x}$ be the $W_{0}$-orbit of $x$. That is

$$
O_{x}=\left\{w x w^{-1} \in X \mid w \in W_{0}\right\} .
$$

For $x \in X^{+}$, define

$$
S_{x}=\sum_{y \in O_{x}} \theta_{y} .
$$

Let $n$ be the rank of $R$ and $x_{1}, x_{2}, \ldots, x_{n}$ be the fundamental dominant weights. Then we have (see [L1, Theorem 8.1] for a proof)

(b) (Bernstein) The center $Z\left(H_{q}\right)$ of $H_{q}$ is a polynomial algebra over $\mathbb{C}$ in $n$-variables, generated by $S_{x_{i}}, i=1,2, \ldots, n$. The elements $S_{x}, x \in$ $X^{+}$, form a $\mathbb{C}$-basis of the center $Z\left(H_{q}\right)$ of $H_{q}$.

Note that $\Theta_{q}$ is a free $Z\left(H_{q}\right)$-module of rank $\left|W_{0}\right|$ (see $[\mathrm{S}]$ ).

1.4. Let $G$ be a simply connected simple algebraic group over $\mathbb{C}$ with root system $R$. Let $T$ be a maximal torus of $G$. Then we can identify $W_{0}$ with $N_{G}(T) / T$ and identify $X$ with $\operatorname{Hom}\left(T, \mathbb{C}^{*}\right)$.

Let $\mathcal{C}$ be a semisimple class of $G$. Choose an element $s$ in $\mathcal{C} \cap T$. The map $\theta_{x} \rightarrow x(s), x \in X$ defines a homomorphism $\phi_{q, s}^{\prime}: \Theta_{q} \rightarrow \mathbb{C}$. It is known that any algebra homomorphism from $Z\left(H_{q}\right)$ to $\mathbb{C}$ is the restriction of $\phi_{q, s}^{\prime}$ for some $s \in T$. We shall denote by $\phi_{q, s}$ the restriction to $Z\left(H_{q}\right)$ of $\phi_{q, s}^{\prime}$. Let $I_{q, s}$ be the two-sided ideal of $H_{q}$ generated by all $S_{x}-\phi_{q, s}\left(S_{x}\right), x \in X^{+}$. Let $H_{q, s}$ be the quotient algebra $H_{q} / I_{q, s}$. Then $H_{q, s}$ is a $\mathbb{C}$-algebra of dimension $\left|W_{0}\right|^{2}$. Since for any $t \in \mathcal{C} \cap T$, we have $H_{q, s}=H_{q, t}$, the algebra $H_{q, s}$ depends only on the semisimple class $\mathcal{C}$. 
We shall say that the central character $\phi_{q, s}$ admits one-dimensional representations if $H_{q, s}$ has one-dimensional representations.

1.5. Let $\mathfrak{g}$ be the Lie algebra of $G$ and $\mathcal{N}$ be the nilpotent cone of $\mathfrak{g}$. For any semisimple element $s$ in $G$, define $\mathcal{N}_{s, q}$ to be the subset of $\mathcal{N}$ given by $\mathcal{N}_{s, q}=\{N \mid N \in \mathcal{N}, \operatorname{Ad}(s) N=q N\}$. For a nilpotent element $N$ in $\mathcal{N}_{s, q}$, let $\mathcal{B}_{N}^{s}$ be the variety consisting of all Borel subalgebras of $\mathfrak{g}$ which contain $N$ and are fixed by $\mathrm{Ad}(s)$. Let $C_{G}(s)$ (resp. $\left.C_{G}(N)\right)$ be the centralizer of $s$ (resp. $N$ ) in $G$. Denote by $A(s, N)$ the component group $C_{G}(s) \cap C_{G}(N) /\left(C_{G}(s) \cap C_{G}(N)\right)^{o}$ of $C_{G}(s) \cap C_{G}(N)$. Then $A(s, N)$ acts on the total complex coefficient Borel-Moore homology group $H_{*}\left(\mathcal{B}_{N}^{s}\right)$. Let $A(s, N)^{\vee}$ be the set of irreducible representations of $A(s, N)$ that appear in $H_{*}\left(\mathcal{B}_{N}^{s}\right)$.

The group $G$ acts on the set $G_{s s}$ of semisimple elements of $G$ by conjugacy and act on the variety $\mathcal{N}$ by adjoint action. So $G$ acts on the set of the union of all $A(s, N)^{\vee}$. If $\sum_{w \in W_{0}} q^{l(w)} \neq 0$, the DeligneLanglands-Lusztig classification says that the isomorphism classes of irreducible representations of $H_{q}$ is in one-to-one correspondence to the $G$-orbits in the set of all triples $(s, N, \rho), s \in G_{s s}, N \in \mathcal{N}, \rho \in$ $A(s, N)^{\vee}$. See $[\mathrm{K}, \mathrm{KL} 2, \mathrm{X} 2]$.

Let $s$ be a semisimple element in $G$. The group $C_{G}(s)$ acts on the variety $\mathcal{N}_{s, q}$ through the adjoint action of $G$. So $C_{G}(s)$ acts on the set of all $A(s, N)^{\vee}, N \in \mathcal{N}_{q, s}$. The Deligne-Langlands-Lusztig classification is equivalent to the following assertion.

(a)If $\sum_{w \in W_{0}} q^{l(w)} \neq 0$, then the isomorphism classes of irreducible representations of $H_{q, s}$ is in one-to-one correspondence to the $C_{G}(s)$-orbits of the pairs $(N, \rho)\left(N \in \mathcal{N}_{q, s}, \rho \in A(s, N)^{\vee}\right)$.

Since the variety $\mathcal{B}_{N}^{s}$ is not empty for any $N \in \mathcal{N}_{q, s}$ [KL2], by (a) we get

(b) The number of isomorphism classes of irreducible representations of $H_{q, s}$ is not less than the number of the $C_{G}(s)$-orbits in $\mathcal{N}_{q, s}$.

1.6. For a root $\alpha$ in $R$, let $e_{\alpha}$ be a non zero element in the root subspace $\mathfrak{g}_{\alpha}$. Let $\alpha_{1}, \ldots, \alpha_{n}$ be the simple roots of $R$. We sometimes use $e_{i}$ for $e_{\alpha_{i}}$. 
For a root $\alpha$ in $R$, let $U_{\alpha}$ be the corresponding one parameter subgroup of $G$ and $u_{\alpha}: \mathbb{C} \rightarrow U_{\alpha}$ be an isomorphism such that $t u_{\alpha}(c) t^{-1}=$ $u_{\alpha}(\alpha(t) c)$ for any $c \in \mathbb{C}$ and $t \in T$.

\section{Group algebra $\mathbb{C}[W]$ of $W$}

In this section we consider the group algebra $\mathbb{C}[W]$, which is identified with $H_{1}$.

Lemma 2.1. Let $s$ be a semisimple element in $T$.

(a) If $H_{1, s}$ has a one-dimensional representation, then $s$ is in the center of $G$, i.e. $\alpha(s)=1$ for simple roots $\alpha$ in $R$.

(b) If $s$ is in the center of $G$, then $H_{1, s}$ has a one-dimensional representation.

Proof. (a) The following argument is provided by the referee. Suppose that $\pi: H_{1} \rightarrow \mathbb{C}$ is a one-dimensional representation of $H_{1}$ (and of $\left.H_{1, s}\right)$. This $\pi$ determines a character of $X$, hence an element $t$ of $T$ conjugate to $s$, by $\pi(x)=x(t)$ for $x \in X$. Since $\pi\left(w x w^{-1}\right)=\pi(x)$ for $x \in X, w \in W_{0}$, we see that $w(t)=t$ for any $w \in W_{0}$. Hence $t$ is in the center of $G$ and $s=t$, (a) is true.

(b) Let $\Omega$ be the subgroup of $W$ consisting of elements of length 0 . Then $\Omega$ is a finite abelian group and is isomorphic to the center of $G$. We identify the character group $\Omega^{*}$ of $\Omega$ with the center of $G$. If $s$ is an element in the center of $G$ (which is identified with $\Omega^{*}$ ), then the one-dimensional representation $\chi: H_{1} \rightarrow \mathbb{C}$ defined by $\chi(w)=1$ for all $w \in W_{a}$ and $\chi(\omega)=s(\omega)$ for any $\omega \in \Omega$ has in fact central character $\phi_{1, s}$. Hence, $H_{1, s}$ has a one-dimensional representation.

The lemma is proved.

Corollary 2.2. The number of central characters $\phi_{1, s}(s \in T)$ which admit one-dimensional representations is equal to the cardinality of the center of $G$.

2.3. Assume that $s$ is in the center. Then $A(s, N)$ is just the component group $A(N)=C_{G}(N) / C_{G}(N)^{\circ}$ of $C_{G}(N)$ for any nilpotent element $N$ in $\mathfrak{g}$. Any Borel subalgebra of $\mathfrak{g}$ is fixed by $\operatorname{Ad}(s)$. So the variety of $\mathcal{B}_{N}^{s}$ is just the variety $\mathcal{B}_{N}$ of all Borel subalgebra of $\mathfrak{g}$ containing $N$. It is known that an irreducible representation of $A(N)$ appears 
in the total Borel-Moore homology of $\mathcal{B}_{N}$ if and only if it appears in the top homology of the $\mathcal{B}_{N}$. Combining the Deligne-Langlands-Lusztig classification for $H_{1}$ (see $\left.1.5(\mathrm{a})\right)$ and Springer's correspondence for $W_{0}$ we get the following fact.

(a) If $s$ is in the center of $G$, then the isomorphism classes of irreducible representations of $H_{1, s}$ is in one-to-one correspondence to the isomorphism classes of irreducible complex representations of $W_{0}$.

The number $\left|\operatorname{Irr}\left(W_{0}\right)\right|$ of isomorphism classes of irreducible complex representations of $W_{0}$ is well known. For convenience, we list them as follows:

Type $A_{n}$ : the number of all partitions of $n+1$,

Type $B_{n}(n \geq 2)$ and $C_{n}(n \geq 3)$ : the number of ordered pairs $(\xi, \eta)$ of partitions $\xi, \eta$ with $|\xi|+|\eta|=n$,

Type $D_{n}(n \geq 4)$ : the number of unordered pairs $(\xi, \eta)$ of partitions $\xi, \eta$ with $|\xi|+|\eta|=n$, and any pairs $(\xi, \eta)$ with $\xi=\eta$ and $|\xi|+|\eta|=n$ is counted twice.

Type $E_{6}, E_{7}, E_{8}: 25,60,112$, respectively

Type $F_{4}: 25$,

Type $G_{2}: 6$.

\section{Some particular semisimple elements in $T$}

In this section we assume that $q \neq 1$. We are interested in the quotient algebras $H_{q, s}$ which have one-dimensional representations.

Lemma 3.1. Assume that $H_{q, s}$ has a one-dimensional representation on which all $T_{r}(r \in S)$ act by the same scalar multiplication. Then in the conjugacy class of $s$ we can find an element $t \in T$ such that $\alpha(t)=q$ for all simple roots $\alpha$.

Proof. The scalar is $q$ or -1 . Using the explicit formulas for reduced expressions of fundamental weights in [L2] we can see that the lemma is true. We illustrate the argument by using type $\tilde{A}_{n}$.

Assume that $R$ is of type $A_{n}$. We number the simple reflections $r_{0}, r_{1}, \ldots, r_{n}$ as usual and simply write $T_{i}$ for $T_{r_{i}}$. Let $\alpha_{1}, \ldots, \alpha_{n}$ be the simple roots and $x_{1}, \ldots, x_{n}$ be the corresponding fundamental weights 
of the weight lattice $X$. According to [L2], for $1 \leq i \leq n$, we have $T_{x_{i}}=T_{\tau^{n+1-i}}\left(T_{n+1-i} T_{n+2-i} \cdots T_{n}\right)\left(T_{n-i} T_{n+1-i} \cdots T_{n-1}\right) \cdots\left(T_{1} T_{2} \cdots T_{i}\right)$, where $\tau \in W$ has length 0 and $\tau r_{0}=r_{1} \tau, \tau r_{1}=r_{2} \tau, \ldots, \tau r_{n}=r_{0} \tau$. Note that $\tau^{n+1}=e$ is the neutral element of $W$.

In $X$ we set $x_{0}=x_{n+1}=0$. Then the length of $x_{i}$ is $i(n+1-i)$. By definition, for $1 \leq i \leq n$, we have

$$
\theta_{\alpha_{i}}=\left(q^{-i(n+1-i)} T_{x_{i}}^{2}\right)\left(q^{-(i+1)(n-i) / 2} T_{x_{i+1}}\right)^{-1}\left(q^{-(i-1)(n+2-i) / 2} T_{x_{i-1}}\right)^{-1} .
$$

If all $T_{i}$ act on a one-dimensional representation of $H_{q, s}$ by scalar $q$, then for all $1 \leq i \leq n, \theta_{\alpha_{i}}$ act on the one-dimensional representation of $H_{q, s}$ by scalar $q$. So the required $t$ exists in this case.

If all $T_{i}$ act on a one-dimensional representation of $H_{q, s}$ by scalar -1 , then for all $1 \leq i \leq n, \theta_{\alpha_{i}}$ act on the one-dimensional representation of $H_{q, s}$ by scalar $q^{-1}$. This means that in the conjugacy class $\mathcal{C}$ of $s$, there exists $t^{\prime} \in \mathcal{C} \cap T$ such that $\alpha_{i}\left(t^{\prime}\right)=q^{-1}$ for all $i=1,2, \ldots, n$. Let $w_{0}$ be the longest element of $W_{0}$ and Set $t=w_{0}\left(t^{\prime}\right)$. Then $t$ is in $\mathcal{C} \cap T$ and $\alpha_{i}(t)=q$ for all $i=1,2, \ldots, n$. The lemma is proved for type $\tilde{A}$. The proof for other types is similar.

Lemma 3.2. Assume that $(q-1) \sum_{w \in W_{0}} q^{l(w)} \neq 0$ and $H_{q, s}$ has a one-dimensional representation on which some $T_{r}(r \in S)$ act by scalar multiplication of $q$ and some $T_{r}(r \in S)$ act by scalar multiplication of -1 . Then in the conjugacy class of $s$ there is no element $t \in T$ such that $\alpha(t)=q$ for all simple roots $\alpha$.

Proof. The root system $R$ must be one of the following types: $B_{n}(n \geq 2), C_{n}(n \geq 3), F_{4}, G_{2}$. We prove the lemma case by case. Let $t \in T$ be such that $\alpha(t)=q$ for all simple roots $\alpha$.

Type $B_{n}(n \geq 2)$. There exist $\varepsilon_{1}, \varepsilon_{2}, \ldots, \varepsilon_{n}$ in $\operatorname{Hom}\left(T, \mathbb{C}^{*}\right)$ such that $\alpha_{i}=\varepsilon_{i}-\varepsilon_{i+1}$ for $i=1, \ldots, n-1$ and $\alpha_{n}=\varepsilon_{n}$. The maximal exponent of $R$ is $2 n-1$ and $\sum_{w \in W_{0}} q^{l(w)}=(q-1)^{-n} \prod_{i=1}^{n}\left(q^{2 i}-1\right)$.

Let $r_{i}$ be the simple reflection corresponding to $\alpha_{i}$ and $r_{0}$ the simple reflection out of $W_{0}$. Assume $T_{r_{1}}, T_{r_{2}}, \ldots, T_{r_{n-1}}$ act on the onedimensional representation by scalar $q$ and $T_{r_{0}}, T_{r_{n}}$ act on it by scalar -1 . Using the explicit formula for $\theta_{x_{i}}$ in [L2] we see that $\alpha_{i}(s)=q$ 
for $i=1,2, \ldots, n-1$ and $\alpha_{n}(s)=q^{-1}$. Therefore $C_{G}(s)$ contains $T, U_{ \pm \varepsilon_{n-1}}, U_{ \pm\left(\varepsilon_{n-2}+\varepsilon_{n}\right)}$.

If the order $o(q)$ is greater than the maximal exponent $2 n-1$, then $t$ is regular and $C_{G}(t)=T$. In this case $s$ and $t$ are not conjugate in $G$.

Now assume that $o(q) \leq 2 n-1$. Our assumption on $q$ implies that $o(q)$ is odd and $n+1 \leq o(q) \leq 2 n-1$. Let $o(q)=n+i$ for some $1 \leq i \leq n-1$. Since $o(q)=n+i$, The centralizer $C_{G}(t)$ of $t$ is generated by $T$ and all $U_{\alpha}$ with $\alpha(t)=1$. It is easy to see that $\alpha(t)=1$ if and only if $\alpha$ is one of the following roots: $\pm\left(\varepsilon_{j}+\varepsilon_{n+2-j-i}\right), 1 \leq j<\frac{n+2-i}{2}$. Noting that all the roots $\varepsilon_{j}+\varepsilon_{n+1-j-i}$ are long roots and $\varepsilon_{n-1}$ is short root, we see that $C_{G}(s)$ and $C_{G}(t)$ are not conjugate in $G$, so $s$ and $t$ are not conjugate in $G$.

Assume $T_{r_{1}}, T_{r_{2}}, \ldots, T_{r_{n-1}}$ act on the one-dimensional representation by scalar -1 and $T_{r_{0}}, T_{r_{n}}$ act on it by scalar $q$. Again using the explicit formula for $\theta_{x_{i}}$ in [L2] we see that $\alpha_{i}(s)=q^{-1}$ for $i=1,2, \ldots, n-1$ and $\alpha_{n}(s)=q$. Let $g \in N_{G}(T)$ be a representative of the longest element of $W_{0}$ and $s^{\prime}=g s g^{-1}$. Then $\alpha_{i}\left(s^{\prime}\right)=q$ for $i=1,2, \ldots, n-1$ and $\alpha_{n}\left(s^{\prime}\right)=q^{-1}$. By the above argument we see that $s^{\prime}$ and $t$ are not conjugate in $G$.

Type $C_{n}(n \geq 3)$. The argument is similar to that for type $B_{n}$.

Type $F_{4}$. Let $V=\mathbb{R}^{4}$ and $\varepsilon_{1}, \ldots, \varepsilon_{4}$ the standard basis of $V$. We may assume that $R$ consists of the following elements:

$\pm \varepsilon_{i}(1 \leq i \leq 4), \quad \pm \varepsilon_{i} \pm \varepsilon_{j}(1 \leq i<j \leq 4)$,

$\frac{1}{2}\left( \pm \varepsilon_{1} \pm \varepsilon_{2} \pm \varepsilon_{3} \pm \varepsilon_{4}\right)$.

The maximal exponent of $R$ is 11 and

$$
\sum_{w \in W_{0}} q^{l(w)}=(q-1)^{-3}(q+1)\left(q^{6}-1\right)\left(q^{8}-1\right)\left(q^{12}-1\right) .
$$

Let $r_{i}$ be the simple reflection corresponding to $\alpha_{i}$ and $r_{0}$ is the reflection out of $W_{0}$. Then $r_{0} r_{4} r_{0}=r_{4} r_{0} r_{4}$. Assume $T_{r_{1}}, T_{r_{2}}$ act on the one-dimensional representation by scalar $q$ and $T_{r_{3}}, T_{r_{4}}, T_{r_{0}}$ act on it by scalar -1 .

According to $[\mathrm{L} 2, \mathrm{p} .646]$, in $H_{q}$ we have

$$
\theta_{x_{4}}=q^{-8} T_{r_{0}} T_{r_{4}} T_{r_{3}} T_{r_{2}} T_{r_{1}} T_{r_{3}} T_{r_{4}} T_{r_{2}} T_{r_{3}} T_{r_{2}} T_{r_{4}} T_{r_{3}} T_{r_{1}} T_{r_{2}} T_{r_{3}} T_{r_{4}},
$$




$$
\begin{gathered}
\theta_{x_{3}} \theta_{x_{4}}^{-1}=q T_{r_{4}}^{-1} \theta_{x_{4}} T_{r_{4}}^{-1}, \\
\theta_{x_{2}} \theta_{x_{3}}^{-1}=q T_{r_{3}}^{-1} \theta_{x_{3}} \theta_{x_{4}}^{-1} T_{r_{3}}^{-1}, \\
\theta_{x_{1}} \theta_{x_{2}}^{-1} \theta_{x_{3}}=q T_{r_{2}}^{-1} \theta_{x_{2}} \theta_{x_{3}}^{-1} T_{r_{2}}^{-1} .
\end{gathered}
$$

So we have $\alpha_{1}(s)=\alpha_{2}(s)=q$ and $\alpha_{3}(s)=\alpha_{4}(s)=q^{-1}$. The centralizer $C_{G}(s)$ of $s$ contains $T, U_{ \pm\left(\alpha_{2}+\alpha_{3}\right)}, U_{ \pm\left(\alpha_{1}+\alpha_{2}+2 \alpha_{3}\right)}, U_{ \pm\left(\alpha_{1}+\alpha_{2}+\alpha_{3}+\alpha_{4}\right)}$, $U_{ \pm\left(\alpha_{1}+2 \alpha_{2}+2 \alpha_{3}+\alpha_{4}\right)}$.

If the order $o(q)$ is greater than 11 , then $t$ is regular and $C_{G}(s)=T$, so $s$ and $t$ are not conjugate.

Now assume that $o(q) \leq 11$. Our assumption on $q$ implies that $o(q)=5,7,9,10,11$. If $o(q)=11$, then $C_{G}(t)$ is generated by $T$ and $U_{ \pm \beta}$, where $\beta$ is the highest root in $R$. If $o(q)=10$, then $C_{G}(t)$ is generated by $T$ and $U_{ \pm\left(\beta-\alpha_{1}\right)}$. If $o(q)=9$, then $C_{G}(t)$ is generated by $T, U_{ \pm\left(\beta-\alpha_{1}-\alpha_{2}\right)}$. If $o(q)=7$, then $C_{G}(t)$ is generated by $T$, $U_{ \pm\left(\beta-\alpha_{1}-\alpha_{2}-2 \alpha_{3}\right)}$. $U_{ \pm\left(\beta-\alpha_{1}-\alpha_{2}-\alpha_{3}-\alpha_{4}\right)}$. If $o(q)=5$, then $C_{G}(t)$ is generated by $T, U_{ \pm\left(\alpha_{1}+2 \alpha_{2}+2 \alpha_{3}\right)}, U_{ \pm\left(\alpha_{2}+2 \alpha_{3}+2 \alpha_{4}\right)}, U_{ \pm\left(\beta-\alpha_{1}\right)}$. In all cases $C_{G}(s)$ and $C_{G}(t)$ are not conjugate in $G$. Hence $s$ and $t$ are not conjugate in $G$.

Assume $T_{r_{1}}, T_{r_{2}}$ act on the one-dimensional representation by scalar -1 and $T_{r_{3}}, T_{r_{4}}, T_{r_{0}}$ act on it by scalar $q$. One can check as above that $\alpha_{1}(s)=\alpha_{2}(s)=q^{-1}$ and $\alpha_{3}(s)=\alpha_{4}(s)=q$. Let $g \in N_{G}(T)$ be a representative of the longest element of $W_{0}$ and $s^{\prime}=g s g^{-1}$. Then $\alpha_{1}\left(s^{\prime}\right)=\alpha_{2}\left(s^{\prime}\right)=q$ and $\alpha_{3}\left(s^{\prime}\right)=\alpha_{4}\left(s^{\prime}\right)=q^{-1}$. By the above discussion we know that $s^{\prime}$ and $t$ are not conjugate in $G$.

Type $G_{2}$. We number the simple reflections $r_{0}, r_{2}, r_{2}$ so that $r_{0} r_{2}=$ $r_{2} r_{0}$. In $W_{0}$ we have $x_{1}=r_{0} r_{1} r_{2} r_{1} r_{2} r_{1}$ and $x_{2}=r_{0} r_{1} r_{2} r_{1} r_{2} r_{0} r_{1} r_{2} r_{1} r_{2}$. In the weight lattice we have $x_{1}=2 \alpha_{1}+\alpha_{2}$ and $x_{2}=3 \alpha_{1}+2 \alpha_{2}$, where $\alpha_{i}$ are the simple roots. If $T_{r_{1}}$ and $T_{r_{0}}$ act on the one-dimensional representation by scalar $q$ and $T_{r_{2}}$ acts it by scalar -1 , then both $\theta_{x_{1}}$ and $\theta_{x_{2}}$ act on it by scalar $q$. So $\alpha_{1}(s)=q$ and $\alpha_{2}(s)=q^{-1}$. Note that $\left(\alpha_{1}+\alpha_{2}\right)(s)=1$. So $C_{G}(s)$ contains $T$ and $U_{ \pm\left(\alpha_{1}+\alpha_{2}\right)}$.

If the order $o(q)$ of $q$ greater than 5 , then $C_{G}(s)=T$. So $s$ and $t$ are not conjugate. If $o(q) \leq 5$, then $o(q)=5,4$ since $\sum_{w \in W_{0}} q^{l(w)} \neq 0$. In these cases $C_{G}(s)$ is generated by $T$ and $U_{ \pm\left(\alpha_{1}+\alpha_{2}\right)}, C_{G}(t)$ is generated 
by $T$ and $U_{ \pm\left(3 \alpha_{1}+\alpha_{2}\right)}$ if $o(q)=4$ or $U_{ \pm\left(3 \alpha_{1}+2 \alpha_{2}\right)}$ if $o(q)=5$. Clearly $C_{G}(s)$ and $C_{G}(t)$ are not conjugate, so $s$ and $t$ are not conjugate.

If $T_{r_{1}}$ and $T_{r_{0}}$ act on the one-dimensional representation by scalar -1 and $T_{r_{2}}$ acts it by scalar $q$, then $\alpha_{1}(s)=q^{-1}$ and $\alpha_{2}(s)=q$. Let $g \in N_{G}(T)$ be a representative of the longest element of $W_{0}$ and $s^{\prime}=g s g^{-1}$. Then $\alpha_{1}\left(s^{\prime}\right)=q$ and $\alpha_{2}\left(s^{\prime}\right)=q^{-1}$. By the above discussion we know that $s^{\prime}$ and $t$ are not conjugate in $G$.

The lemma is proved.

Corollary 3.3. Assume that the root system $R$ is not simply laced. If $(q-1) \sum_{w \in W_{0}} q^{l(w)} \neq 0$, then the number of central characters $\phi_{q, s}(s \in$ $T$ ) which admit one-dimensional representations is twice the cardinality of the center of $G$.

Proof. Let $s \in T$ be such that $\alpha(s)=q$ for all simple roots $\alpha$. It is easy to check that the map $T_{r} \theta_{x} \rightarrow q x(s)$ for any simple reflection $r$ in $W_{0}$ and $x \in X$ defines an algebra homomorphism $H_{q} \rightarrow \mathbb{C}$. Hence, the central character $\phi_{q, s}$ admits one-dimensional representations of $H_{q}$ on which all $T_{i}$ act by the same scalar. Since $(q-1) \sum_{w \in W_{0}} q^{l(w)} \neq 0$, we have $q \neq-1$. So we can find $t \in T$ such that the central character $\phi_{q, t}$ admits one-dimensional representations of $H_{q}$ on which some $T_{i}$ act by scalar $q$ and some $T_{i}$ act by scalar -1 . By the calculations in the proof of Lemma 3.2, it is no harm to assume that $\alpha(t)=q$ for short simple roots $\alpha$ and $\alpha(t)=q^{-1}$ for long simple roots $\alpha$.

By Lemma 3.2, $s$ and $t$ are not conjugate in $G$ if $(q-1) \sum_{w \in W_{0}} q^{l(w)} \neq$ 0 .

For types $F_{4}$ and $G_{2}$, the center of $G$ is trivial and the weight lattice equals the root lattice. Hence there are exactly two central characters of $H_{q}$ which admit one-dimensional representations if ( $q-$ 1) $\sum_{w \in W_{0}} q^{l(w)} \neq 0$.

Now assume that $R$ is of type $B_{n}(n \geq 2)$ or $C_{n}(n \geq 3)$. Let $s^{\prime} \in T$. Assume that the central character $\phi_{q, s^{\prime}}$ admits one-dimensional representations of $H_{q}$ on which all $T_{i}$ act by the same scalar. By Lemma 3.1 , in the conjugacy class of $s^{\prime}$ we can find $s^{\prime \prime} \in T$ such that $\alpha\left(s^{\prime \prime}\right)=q$ for all simple roots $\alpha$. Then $s^{\prime \prime} s^{-1}=c$ is in the center of $G$. We 
need show that $s^{\prime \prime}$ and $s$ are not conjugate in $G$ if $c$ is non-trivial and $(q-1) \sum_{w \in W_{0}} q^{l(w)} \neq 0$.

First consider type $B_{n}$. In this case $G=\operatorname{Spin}_{2 n+1}(\mathbb{C})$ and $S O_{2 n+1}(\mathbb{C})$ is its quotient group. Clearly, $s^{\prime \prime}$ and $s$ have the same image in $S_{2 n+1}(\mathbb{C})$. It is no harm to assume the image is

$$
\operatorname{diag}\left(1, q^{n}, \ldots, q^{2}, q^{1}, q^{-n}, \ldots, q^{-2}, q^{-1}\right) .
$$

If $s^{\prime \prime}$ and $s$ are conjugate in $G$, we must have $q=q^{i}$ for some $2 \leq i \leq n$ or $q=q^{-j}$ for some $1 \leq j \leq n$. If $j \neq n$, then $(q-1) \sum_{w \in W_{0}} q^{l(w)}=0$. If $j=n$, then $q^{n+1}=1$. We also have $q^{\frac{n(n+1)}{2}}=-1$ in this case since $c$ is non-trivial. This forces that $n+1$ is even and $\sum_{w \in W_{0}} q^{l(w)}=0$. So $s^{\prime \prime}$ and $s$ are not conjugate if $(q-1) \sum_{w \in W_{0}} q^{l(w)} \neq 0$.

Now assume that $R$ is of type $C_{n}$. Then $G=S p_{2 n}(\mathbb{C})$. If $c$ is nontrivial, then we may assume that $s, s^{\prime \prime}$ are the following two elements:

$$
\begin{gathered}
\operatorname{diag}\left(q^{\frac{2 n-1}{2}}, \ldots, q^{\frac{3}{2}}, q^{\frac{1}{2}}, q^{-\frac{2 n-1}{2}}, \ldots, q^{-\frac{3}{2}}, q^{-\frac{1}{2}}\right) \\
\operatorname{diag}\left(-q^{\frac{2 n-1}{2}}, \ldots,-q^{\frac{3}{2}},-q^{\frac{1}{2}},-q^{-\frac{2 n-1}{2}}, \ldots,-q^{-\frac{3}{2}},-q^{-\frac{1}{2}}\right) .
\end{gathered}
$$

If $s^{\prime \prime}$ and $s$ are conjugate, then $q^{\frac{1}{2}}=-q^{\frac{1}{2}+i}$ for some $n-1 \geq i \geq 1$ or $q^{\frac{1}{2}}=-q^{-\frac{1}{2}-j}$ for some $n-1 \geq j \geq 0$. So, $q^{i}=-1$ or $q^{1+j}=-1$. This contradicts that $(q-1) \sum_{w \in W_{0}} q^{l(w)} \neq 0$. Hence, $s^{\prime \prime}$ and $s$ are not conjugate in $G$.

Assume that the central character $\phi_{q, t^{\prime}}$ admits one-dimensional representations of $H_{q}$ on which some $T_{i}$ act by the scalar $q$ and some $T_{i}$ act by the scalar -1 . By the calculations in the proof of Lemma 3.2, in the conjugacy class of $t^{\prime}$ we can find $t^{\prime \prime} \in T$ such that $\alpha\left(t^{\prime \prime}\right)=q$ for all simple short roots $\alpha$ and $\alpha\left(t^{\prime \prime}\right)=q^{-1}$ for all simple long roots $\alpha$. Then $t^{\prime \prime} t^{-1}=c$ is in the center of $G$. As above, we can show that $t^{\prime \prime}$ and $t$ are not conjugate in $G$ if $c$ is non-trivial and $(q-1) \sum_{w \in W_{0}} q^{l(w)} \neq 0$.

The corollary is proved.

Lemma 3.4. Assume that the order of $q$ is greater than the maximal exponent of the root system $R$. Let $s \in T$ be such that $\alpha(s)=q$ for all simple roots $\alpha$ in $R$. Then

(a) The number of $C_{G}(s)$-orbits of the variety $\mathcal{N}_{q, s}$ is equal to $2^{n}$. 
(b) For any $N \in \mathcal{N}_{q, s}$, the group $A(s, N)$ acts on $H_{*}\left(\mathcal{B}_{N}^{s}\right)$ trivially, so $A(s, N)^{\vee}$ contains only the trivial representation of $A(s, N)$.

(c) The number of the isomorphism classes of irreducible representations of $H_{q, s}$ is $2^{n}$.

Proof. Since the order of $q$ is greater than the maximal exponent of the root system $R$, we see that $s$ is regular, so $C_{G}(s)=T$. Moreover, we have $\mathcal{N}_{q, s}=\left\{\sum_{1 \leq i \leq n} a_{i} e_{i} \mid a_{i} \in \mathbb{C}\right\}$. For a subset $I$ of $\{1,2, \ldots, n\}$, set $e_{I}=\sum_{i \in I} e_{i}$. (We understand that $e_{I}=0$ if $I$ is the empty set.) Then any element in $\mathcal{N}_{q, s}$ is $C_{G}(s)$-conjugate to some $e_{I}$. Clearly if $I \neq J$, the $e_{I}$ and $e_{J}$ are not in the same $C_{G}(s)$-orbit. Part (a) is proved.

Since $s$ is regular, the variety $\mathcal{B}^{s}$ of Borel subalgebras of $\mathfrak{g}$ fixed by $\operatorname{Ad}(s)$ is exactly the variety $\mathcal{B}^{T}$ of Borel subalgebras of $\mathfrak{g}$ fixed by $\operatorname{Ad} T$. Now $C_{G}(s)=T$, so for any $N \in \mathcal{N}_{q, s}$, the group $C_{G}(s) \cap C_{G}(N)$ acts trivially on $\mathcal{B}_{N}^{s}$. Part (b) follows.

Part (c) follows from (a) and (b). The lemma is proved.

3.5. In the rest part of this section $R$ is assumed to be simply laced and $H_{q, s}$ is required to satisfy the assumption of Lemma 3.1. We hope to know the number of the $C_{G}(s)$-orbits in $\mathcal{N}_{q, s}$, provided that $(q-$ 1) $\sum_{w \in W_{0}} q^{l(w)} \neq 0$ and the order $o(q)$ of $q$ is not greater than the maximal exponent of $R$. The following fact of Lusztig [L3] will be useful.

(a) Assume $x \in \mathfrak{g}$ and $\operatorname{Ad}(s)(x)=q x$. If $(q-1) \sum_{w \in W_{0}} q^{l(w)} \neq 0$, then $x \in \mathcal{N}_{q, s}$.

Since $\alpha(s)=q$ for all simple roots $\alpha$ in $R$, by (a) we get

(b) $\mathcal{N}_{q, s}$ is a linear space spanned by all $e_{\beta}$ with $\beta(s)=q$ and is naturally a $C_{G}(s)$-module.

Since $T \subset C_{G}(s)$, any $C_{G}(s)$-submodule of $\mathcal{N}_{q, s}$ is spanned by some $e_{\beta}$ with $\beta(s)=q$. Since $C_{G}(s)$ is reductive, $\mathcal{N}_{q, s}$ is the direct sum of some irreducible $C_{G}(s)$-submodules of $\mathcal{N}_{q, s}$.

We discuss, on a case by case basis, the number of $C_{G}(s)$-orbits in $\mathcal{N}_{q, s}$. To do this, we first decompose $\mathcal{N}_{q, s}$ into the direct sum of some irreducible $C_{G}(s)$-submodules. Let $\beta \in R$ be such that $\beta(s)=1$ and $u_{\beta}(\xi)(\xi \in \mathbb{C}$, see 1.6 for definition) an element in the one parameter 
subgroup $U_{\beta}$ of $G$. Then we may assume that $u_{\beta}(\xi)$ acts on $\mathfrak{g}$ (hence $\left.\mathcal{N}_{q, s}\right)$ through $\exp \left(\xi \operatorname{ad}\left(e_{\beta}\right)\right)$. This idea is useful in determining the submodule structure of $\mathcal{N}_{q, s}$. Recall that we identify the weight lattice $X$ of $R$ with $\operatorname{Hom}\left(T, \mathbb{C}^{*}\right)$.

Type $A_{n}$ The maximal exponent is $n$ and $\sum_{w \in W_{0}} q^{l(w)}=(q-1)^{-n} \prod_{i=2}^{n+1}\left(q^{i}-\right.$ $1)$. There are no complex numbers $q \neq 1$ such that its order is not greater than $n$ and $\sum_{w \in W_{0}} q^{l(w)} \neq 0$.

Type $D_{n}(n \geq 4)$ There exist $\varepsilon_{1}, \varepsilon_{2}, \ldots, \varepsilon_{n}$ in $\operatorname{Hom}\left(T, \mathbb{C}^{*}\right)$ such that $\alpha_{i}=$ $\varepsilon_{i}-\varepsilon_{i+1}$ for $i=1, \ldots, n-1$ and $\alpha_{n}=\varepsilon_{n-1}+\varepsilon_{n}$. The maximal exponent of $R$ is $2 n-3$ and $\sum_{w \in W_{0}} q^{l(w)}=(q-1)^{-n}\left(q^{n}-1\right) \prod_{i=1}^{n-1}\left(q^{2 i}-1\right)$. The assumption on $q$ implies that $o(q)$ is odd and $n+1 \leq o(q) \leq 2 n-3$. Let $o(q)=n+i$ for some $1 \leq i \leq n-3$.

By assumption, $\alpha_{i}(s)=q$. Since $o(q)=n+i$, we see the other roots satisfying $\alpha(s)=q$ are the following: $\varepsilon_{j}+\varepsilon_{n-1-j-i},-\varepsilon_{k}-\varepsilon_{n+1-k-i}$, with $1 \leq j<\frac{n-i-1}{2}$ and $1 \leq k<\frac{n+1-i}{2}$.

It is easy to check that $\alpha(s)=1$ if and only if $\alpha$ is one of the following roots: $\pm\left(\varepsilon_{j}+\varepsilon_{n-j-i}\right), 1 \leq j<\frac{n-i}{2}$.

If $i=n-3$, then $C_{G}(s)$ is generated by $T$ and $U_{ \pm\left(\varepsilon_{1}+\varepsilon_{2}\right)}$. The linear space $\mathcal{N}_{q, s}$ is spanned by $e_{\varepsilon_{1}-\varepsilon_{2}}, e_{\varepsilon_{2}-\varepsilon_{3}}, \ldots, e_{\varepsilon_{n-1}-\varepsilon_{n}}, e_{\varepsilon_{n-1}+\varepsilon_{n}}$ and $e_{-\varepsilon_{1}-\varepsilon_{3}}$. It is easy to see that $e_{\varepsilon_{2}-\varepsilon_{3}}$ and $e_{-\varepsilon_{1}-\varepsilon_{3}}$ span a $C_{G}(s)$-submodule $M_{2}$ of $\mathcal{N}_{q, s}$. For any $1 \leq j \neq 2 \leq n-1$, the element $e_{\varepsilon_{j}-\varepsilon_{j+1}}$ spans a one-dimensional $C_{G}(s)$-submodule $M_{j}$ of $\mathcal{N}_{q, s}$. The space $\mathcal{N}_{q, s}$ is the direct sum of the submodules $M_{1}, M_{2}, \ldots, M_{n}$ and each $M_{j}$ has two $C_{G}(s)$-orbits. Therfore the number of $C_{G}(s)$-orbits in $\mathcal{N}_{q, s}$ is $2^{n}$.

Now assume that $i \leq n-5$. The following elements span a $C_{G}(s)$ submodule $M_{j}$ of $\mathcal{N}_{q, s}: e_{\varepsilon_{j}-\varepsilon_{j+1}}, e_{-\varepsilon_{j+1}-\varepsilon_{n-j-i}}, e_{\varepsilon_{j}+\varepsilon_{n-j-1-i}}, e_{\varepsilon_{n-j-1-i}-\varepsilon_{n-j-i}}$ for $j=1,2, \ldots, \frac{n-i-3}{2}$. The elements $e_{\varepsilon_{j}-\varepsilon_{j+1}}$ and $e_{-\varepsilon_{j-1}-\varepsilon_{j+1}}$ span a two-dimensional $C_{G}(s)$-submodule $M_{j}^{\prime}$ of $\mathcal{N}_{q, s}$ for $j=\frac{n-i+1}{2}$. The element $e_{\varepsilon_{j}-\varepsilon_{j+1}}$ spans a one-dimensional $C_{G}(s)$-submodule $M_{j}^{\prime}$ of $\mathcal{N}_{q, s}$ for $j=\frac{n-i-1}{2}, n-i, n-i+1, \ldots, n-1$. Also the element $e_{\varepsilon_{n-1}+\varepsilon_{n}}$ spans a one-dimensional $C_{G}(s)$-submodule $M_{n}^{\prime}$ of $\mathcal{N}_{q, s}$ Clearly $\mathcal{N}_{q, s}$ is the direct sum of all the submodules $M_{j}, M_{k}^{\prime}$. One may check easily that each 
$M_{j}$ has three $C_{G}(s)$-orbits and each $M_{k}^{\prime}$ has two $C_{G}(s)$-orbits. So the number of $C_{G}(s)$-orbits in $\mathcal{N}_{q, s}$ is not less than $2^{i+3} \cdot 3^{\frac{n-i-3}{2}}$.

Type $E_{6}$ We number the simple roots as in [B]. One has

$$
\sum_{w \in W_{0}} q^{l(w)}=\frac{\left(q^{2}-1\right)\left(q^{5}-1\right)\left(q^{6}-1\right)\left(q^{8}-1\right)\left(q^{9}-1\right)\left(q^{12}-1\right)}{(q-1)^{6}} .
$$

The maximal exponent is 11 . The assumption on $q$ implies that $o(q)=$ $7,10,11$.

If $o(q)=7$, then $C_{G}(s)$ is generated by $T, U_{ \pm \beta_{i}}, i=1,2,3$, here $\beta_{1}=\alpha_{1}+\alpha_{2}+2 \alpha_{3}+2 \alpha_{4}+\alpha_{5}, \beta_{2}=\alpha_{2}+\alpha_{3}+2 \alpha_{4}+2 \alpha_{5}+\alpha_{6}, \beta_{3}=$ $\alpha_{1}+\alpha_{2}+\alpha_{3}+2 \alpha_{4}+\alpha_{5}+\alpha_{6}$. If $\alpha$ is not simple root, then $\alpha(s)=q$ if and only if $\alpha$ is one of the following roots: $\gamma_{1}=\alpha_{1}+\alpha_{2}+2 \alpha_{3}+2 \alpha_{4}+\alpha_{5}+\alpha_{6}$, $\gamma_{2}=\alpha_{1}+\alpha_{2}+\alpha_{3}+2 \alpha_{4}+2 \alpha_{5}+\alpha_{6},-\gamma_{3}=-\left(\alpha_{1}+\alpha_{2}+\alpha_{3}+2 \alpha_{4}+\alpha_{5}\right)$, $-\gamma_{4}=-\left(\alpha_{2}+\alpha_{3}+2 \alpha_{4}+\alpha_{5}+\alpha_{6}\right),-\gamma_{5}=-\left(\alpha_{1}+\alpha_{2}+\alpha_{3}+\alpha_{4}+\alpha_{5}+\alpha_{6}\right)$. It is easy to check the following facts:

(1) $e_{\alpha_{1}}, e_{\gamma_{2}}, e_{\alpha_{5}}, e_{-\gamma_{4}}$ span a $C_{G}(s)$-submodule $M_{1}$ of $\mathcal{N}_{q, s}$,

(2) $e_{\alpha_{3}}, e_{\gamma_{1}}, e_{\alpha_{6}}, e_{-\gamma_{3}}$ span a $C_{G}(s)$-submodule $M_{2}$ of $\mathcal{N}_{q, s}$,

(3) $e_{\alpha_{4}}, e_{-\gamma_{5}}$ span a $C_{G}(s)$-submodule $M_{3}$ of $\mathcal{N}_{q, s}$,

(4) $e_{\alpha_{2}}$ spans a $C_{G}(s)$-submodule $M_{4}$ of $\mathcal{N}_{q, s}$.

Clearly $\mathcal{N}_{q, s}$ is the direct sum of all $M_{i}$. Noting that $M_{i}$ has three $C_{G}(s)$-orbits for $i=1,2$ and $M_{j}$ has two $C_{G}(s)$-orbits for $j=3,4$, we see that the number of $C_{G}(s)$-orbits in $\mathcal{N}_{q, s}$ is not less than 36 .

If $o(q)=10$ or 11 , then we can see easily that the number of $C_{G}(s)$ orbits in $\mathcal{N}_{q, s}$ is $2^{6}$.

Type $E_{7}$ We number the simple roots as in [B]. One has

$$
\sum_{w \in W_{0}} q^{l(w)}=\frac{\left(q^{2}-1\right)\left(q^{6}-1\right)\left(q^{8}-1\right)\left(q^{10}-1\right)\left(q^{12}-1\right)\left(q^{14}-1\right)\left(q^{18}-1\right)}{(q-1)^{7}} .
$$

The maximal exponent is 17 . The assumption on $q$ implies that $o(q)=$ $11,13,15,16,17$.

If $o(q)=11$, then $C_{G}(s)$ is generated by $T, U_{ \pm \beta_{i}}, i=1,2,3$, here $\beta_{1}=\alpha_{1}+2 \alpha_{2}+2 \alpha_{3}+3 \alpha_{4}+2 \alpha_{5}+\alpha_{6}, \beta_{2}=\alpha_{1}+\alpha_{2}+2 \alpha_{3}+2 \alpha_{4}+$ $2 \alpha_{5}+2 \alpha_{6}+\alpha_{7}, \beta_{3}=\alpha_{1}+\alpha_{2}+2 \alpha_{3}+3 \alpha_{4}+2 \alpha_{5}+\alpha_{6}+\alpha_{7}$. If $\alpha$ is not simple root, then $\alpha(s)=q$ if and only if $\alpha$ is one of the following roots: $\gamma_{1}=\alpha_{1}+\alpha_{2}+2 \alpha_{3}+3 \alpha_{4}+2 \alpha_{5}+2 \alpha_{6}+\alpha_{7}, \gamma_{2}=\alpha_{1}+2 \alpha_{2}+$ 
$2 \alpha_{3}+3 \alpha_{4}+2 \alpha_{5}+\alpha_{6}+\alpha_{7},-\gamma_{3}=-\left(\alpha_{1}+\alpha_{2}+2 \alpha_{3}+3 \alpha_{4}+2 \alpha_{5}+\alpha_{6}\right)$, $-\gamma_{4}=-\left(\alpha_{1}+\alpha_{2}+2 \alpha_{3}+2 \alpha_{4}+2 \alpha_{5}+\alpha_{6}+\alpha_{7}\right),-\gamma_{5}=-\left(\alpha_{1}+\alpha_{2}+\right.$ $\left.\alpha_{3}+2 \alpha_{4}+2 \alpha_{5}+2 \alpha_{6}+\alpha_{7}\right)$. It is easy to check the following facts:

(5) $e_{\alpha_{2}}, e_{\gamma_{2}}, e_{\alpha_{7}}, e_{-\gamma_{4}}$ span a $C_{G}(s)$-submodule $M_{2}$ of $\mathcal{N}_{q, s}$,

(6) $e_{\alpha_{4}}, e_{\gamma_{1}}, e_{\alpha_{6}}, e_{-\gamma_{4}}$ span a $C_{G}(s)$-submodule $M_{4}$ of $\mathcal{N}_{q, s}$,

(7) $e_{\alpha_{3}}, e_{-\gamma_{5}}$ span a $C_{G}(s)$-submodule $M_{3}$ of $\mathcal{N}_{q, s}$,

(8) For $i=1$ or 5 , the element $e_{\alpha_{i}}$ spans a $C_{G}(s)$-submodule $M_{i}$ of $\mathcal{N}_{q, s}$

Clearly $\mathcal{N}_{q, s}$ is the direct sum of all $M_{i}$. Noting that $M_{i}$ has three $C_{G}(s)$-orbits for $i=2,4$ and $M_{j}$ has two $C_{G}(s)$-orbits for $j=1,3,5$, we see that the number of $C_{G}(s)$-orbits in $\mathcal{N}_{q, s}$ is not less than $2^{3} \times 3^{2}=72$.

If $o(q)=13$, then $C_{G}(s)$ is generated by $T, U_{ \pm \sigma_{i}}, i=1,2$, here $\sigma_{1}=\alpha_{1}+\alpha_{2}+2 \alpha_{3}+3 \alpha_{4}+3 \alpha_{5}+2 \alpha_{6}+\alpha_{7}, \sigma_{2}=\alpha_{1}+2 \alpha_{2}+2 \alpha_{3}+3 \alpha_{4}+$ $2 \alpha_{5}+2 \alpha_{6}+\alpha_{7}$. If $\alpha$ is not simple root, then $\alpha(s)=q$ if and only if $\alpha$ is one of the following roots: $\tau_{1}=\alpha_{1}+2 \alpha_{2}+2 \alpha_{3}+3 \alpha_{4}+3 \alpha_{5}+2 \alpha_{6}+\alpha_{7}$, $-\tau_{2}=-\left(\alpha_{1}+\alpha_{2}+2 \alpha_{3}+3 \alpha_{4}+2 \alpha_{5}+2 \alpha_{6}+\alpha_{7}\right),-\tau_{3}=-\left(\alpha_{1}+2 \alpha_{2}+\right.$ $\left.2 \alpha_{3}+3 \alpha_{4}+2 \alpha_{5}+\alpha_{6}+\alpha_{7}\right)$. It is easy to check the following facts:

(9) $e_{\alpha_{2}}, e_{\tau_{1}}, e_{\alpha_{5}}, e_{-\tau_{2}}$ span a $C_{G}(s)$-submodule $M_{2}$ of $\mathcal{N}_{q, s}$,

(10) $e_{\alpha_{6}}, e_{-\tau_{3}}$ span a $C_{G}(s)$-submodule $M_{6}$ of $\mathcal{N}_{q, s}$,

(11) The element $e_{\alpha_{i}}$ spans a $C_{G}(s)$-submodule $M_{i}$ of $\mathcal{N}_{q, s}$ for $i=$ $1,3,4,7$.

Clearly $\mathcal{N}_{q, s}$ is the direct sum of all $M_{i}$. Noting that $M_{2}$ has three $C_{G}(s)$-orbits and $M_{j}$ has two $C_{G}(s)$-orbits for $j=1,3,4,6,7$, we see that the number of $C_{G}(s)$-orbits in $\mathcal{N}_{q, s}$ is not less than $2^{5} \times 3=96$.

If $o(q)=15,16,17$, then we can see easily that the number of $C_{G}(s)$-orbits in $\mathcal{N}_{q, s}$ is $2^{7}$.

Type $E_{8}$ This is the most complicated case. We number the simple roots as in $[\mathrm{B}]$. One has

$$
\sum_{w \in W_{0}} q^{l(w)}=\frac{\left(q^{2}-1\right)\left(q^{8}-1\right)\left(q^{12}-1\right)\left(q^{14}-1\right)\left(q^{18}-1\right)\left(q^{20}-1\right)\left(q^{24}-1\right)\left(q^{30}-1\right)}{(q-1)^{8}} .
$$

The maximal exponent is 29. The assumption on $q$ implies that $o(q)=$ $11,13,16,17,19,21,22,23,25,26,27,28,29$.

If $o(q)=11$, then $C_{G}(s)$ is generated by $T, U_{ \pm \beta_{i}}, 1 \leq i \leq 6$, here 


$$
\begin{aligned}
& \beta_{1}=\alpha_{1}+2 \alpha_{2}+2 \alpha_{3}+3 \alpha_{4}+2 \alpha_{5}+\alpha_{6}, \\
& \beta_{2}=\alpha_{1}+\alpha_{2}+2 \alpha_{3}+2 \alpha_{4}+2 \alpha_{5}+2 \alpha_{6}+\alpha_{7}, \\
& \beta_{3}=\alpha_{1}+\alpha_{2}+2 \alpha_{3}+3 \alpha_{4}+2 \alpha_{5}+\alpha_{6}+\alpha_{7}, \\
& \beta_{4}=\alpha_{1}+\alpha_{2}+2 \alpha_{3}+2 \alpha_{4}+2 \alpha_{5}+\alpha_{6}+\alpha_{7}+\alpha_{8}, \\
& \beta_{5}=\alpha_{2}+\alpha_{3}+2 \alpha_{4}+2 \alpha_{5}+2 \alpha_{6}+2 \alpha_{7}+\alpha_{8}, \\
& \beta_{6}=\alpha_{1}+\alpha_{2}+\alpha_{3}+2 \alpha_{4}+2 \alpha_{5}+2 \alpha_{6}+\alpha_{7}+\alpha_{8}, \\
& \beta_{7}=\beta_{1}+\beta_{5}=\alpha_{1}+3 \alpha_{2}+3 \alpha_{3}+5 \alpha_{4}+4 \alpha_{5}+3 \alpha_{6}+2 \alpha_{7}+\alpha_{8}, \\
& \beta_{8}=\beta_{3}+\beta_{6}=2 \alpha_{1}+2 \alpha_{2}+3 \alpha_{3}+5 \alpha_{4}+4 \alpha_{5}+3 \alpha_{6}+2 \alpha_{7}+\alpha_{8} .
\end{aligned}
$$

If $\alpha$ is not simple root, then $\alpha(s)=q$ if and only if $\alpha$ is one of the following roots:

$$
\begin{aligned}
& \gamma_{1}=\alpha_{1}+\alpha_{2}+2 \alpha_{3}+3 \alpha_{4}+2 \alpha_{5}+2 \alpha_{6}+\alpha_{7}, \\
& \gamma_{2}=\alpha_{1}+2 \alpha_{2}+2 \alpha_{3}+3 \alpha_{4}+2 \alpha_{5}+\alpha_{6}+\alpha_{7} \\
& -\gamma_{3}=-\left(\alpha_{1}+\alpha_{2}+2 \alpha_{3}+3 \alpha_{4}+2 \alpha_{5}+\alpha_{6}\right) \\
& -\gamma_{4}=-\left(\alpha_{1}+\alpha_{2}+2 \alpha_{3}+2 \alpha_{4}+2 \alpha_{5}+\alpha_{6}+\alpha_{7}\right) \\
& -\gamma_{5}=-\left(\alpha_{1}+\alpha_{2}+\alpha_{3}+2 \alpha_{4}+2 \alpha_{5}+2 \alpha_{6}+\alpha_{7}\right) \\
& -\gamma_{6}=-\left(\alpha_{2}+\alpha_{3}+2 \alpha_{4}+2 \alpha_{5}+2 \alpha_{6}+\alpha_{7}+\alpha_{8}\right) \\
& -\gamma_{7}=-\left(\alpha_{1}+\alpha_{2}+2 \alpha_{3}+2 \alpha_{4}+\alpha_{5}+2 \alpha_{6}+\alpha_{7}+\alpha_{8}\right) \\
& -\gamma_{8}=-\left(\alpha_{1}+\alpha_{2}+\alpha_{3}+2 \alpha_{4}+2 \alpha_{5}+\alpha_{6}+\alpha_{7}+\alpha_{8}\right) \\
& \gamma_{9}=\alpha_{1}+\alpha_{2}+2 \alpha_{3}+3 \alpha_{4}+2 \alpha_{5}+\alpha_{6}+\alpha_{7}+\alpha_{8}, \\
& \gamma_{10}=\alpha_{1}+\alpha_{2}+2 \alpha_{3}+2 \alpha_{4}+2 \alpha_{5}+2 \alpha_{6}+\alpha_{7}+\alpha_{8}, \\
& \gamma_{11}=\alpha_{1}+\alpha_{2}+\alpha_{3}+2 \alpha_{4}+2 \alpha_{5}+2 \alpha_{6}+2 \alpha_{7}+\alpha_{8}, \\
& \gamma_{12}=2 \alpha_{1}+3 \alpha_{2}+3 \alpha_{3}+5 \alpha_{4}+4 \alpha_{5}+3 \alpha_{6}+2 \alpha_{7}+\alpha_{8}, \\
& \gamma_{13}=2 \alpha_{1}+2 \alpha_{2}+4 \alpha_{3}+5 \alpha_{4}+4 \alpha_{5}+3 \alpha_{6}+2 \alpha_{7}+\alpha_{8}, \\
& -\gamma_{14}=-\left(\alpha_{1}+2 \alpha_{2}+3 \alpha_{3}+5 \alpha_{4}+4 \alpha_{5}+3 \alpha_{6}+2 \alpha_{7}+\alpha_{8}\right), \\
& -\gamma_{15}=-\left(2 \alpha_{1}+2 \alpha_{2}+3 \alpha_{3}+4 \alpha_{4}+4 \alpha_{5}+3 \alpha_{6}+2 \alpha_{7}+\alpha_{8}\right),
\end{aligned}
$$

It is easy to check the following fact:

(12) The ten elements $e_{\alpha_{1}}, e_{\gamma_{11}}, e_{\alpha_{7}}, e_{\gamma_{2}}, e_{-\gamma_{6}}, e_{-\gamma_{3}}, e_{\alpha_{2}}, e_{\gamma_{12}}, e_{\gamma_{13}}$, $e_{-\gamma_{14}}$ span a $C_{G}(s)$-submodule $M_{1}$ of $\mathcal{N}_{q, s}$,

(13) The eleven elements $e_{\alpha_{3}}, e_{\gamma_{10}}, e_{-\gamma_{5}}, e_{\alpha_{6}}, e_{\alpha_{8}}, e_{-\gamma_{8}}, e_{-\gamma_{15}}, e_{\gamma_{1}}$, $e_{\alpha_{4}}, e_{\gamma_{9}}, e_{-\gamma_{4}}$ span a $C_{G}(s)$-submodule $M_{2}$ of $\mathcal{N}_{q, s}$,

(14) $e_{\alpha_{5}}, e_{-\gamma_{7}}$ span a $C_{G}(s)$-submodule $M_{3}$ of $\mathcal{N}_{q, s}$.

Clearly $\mathcal{N}_{q, s}$ is the direct sum of all $M_{i}$, but it is not easy to see the number of $C_{G}(s)$-orbits of $\mathcal{N}_{q, s}$ in this case.

If $o(q)=13$, then $C_{G}(s)$ is generated by $T, U_{ \pm \sigma_{i}}, i=1,2$, here 


$$
\begin{aligned}
& \sigma_{1}=\alpha_{1}+\alpha_{2}+2 \alpha_{3}+3 \alpha_{4}+3 \alpha_{5}+2 \alpha_{6}+\alpha_{7}, \\
& \sigma_{2}=\alpha_{1}+2 \alpha_{2}+2 \alpha_{3}+3 \alpha_{4}+2 \alpha_{5}+2 \alpha_{6}+\alpha_{7}, \\
& \sigma_{3}=\alpha_{1}+2 \alpha_{2}+3 \alpha_{3}+2 \alpha_{4}+2 \alpha_{5}+\alpha_{6}+2 \alpha_{7}+\alpha_{8}, \\
& \sigma_{4}=\alpha_{1}+\alpha_{2}+2 \alpha_{3}+3 \alpha_{4}+2 \alpha_{5}+2 \alpha_{6}+\alpha_{7}+\alpha_{8}, \\
& \sigma_{5}=\alpha_{1}+\alpha_{2}+2 \alpha_{3}+2 \alpha_{4}+2 \alpha_{5}+2 \alpha_{6}+2 \alpha_{7}+\alpha_{8}, \\
& \sigma_{6}=\sigma_{1}+\sigma_{3}=2 \alpha_{1}+3 \alpha_{2}+4 \alpha_{3}+6 \alpha_{4}+5 \alpha_{5}+3 \alpha_{6}+2 \alpha_{7}+\alpha_{8} .
\end{aligned}
$$

If $\alpha$ is not simple root, then $\alpha(s)=q$ if and only if $\alpha$ is one of the following roots:

$$
\begin{aligned}
& \tau_{1}=\alpha_{1}+2 \alpha_{2}+2 \alpha_{3}+3 \alpha_{4}+3 \alpha_{5}+2 \alpha_{6}+\alpha_{7}, \\
& -\tau_{2}=-\left(\alpha_{1}+\alpha_{2}+2 \alpha_{3}+3 \alpha_{4}+2 \alpha_{5}+2 \alpha_{6}+\alpha_{7}\right), \\
& -\tau_{3}=-\left(\alpha_{1}+2 \alpha_{2}+2 \alpha_{3}+3 \alpha_{4}+2 \alpha_{5}+\alpha_{6}+\alpha_{7}\right), \\
& -\tau_{4}=-\left(\alpha_{1}+\alpha_{2}+2 \alpha_{3}+3 \alpha_{4}+2 \alpha_{5}+\alpha_{6}+\alpha_{7}+\alpha_{8}\right), \\
& -\tau_{5}=-\left(\alpha_{1}+\alpha_{2}+2 \alpha_{3}+2 \alpha_{4}+2 \alpha_{5}+2 \alpha_{6}+\alpha_{7}+\alpha_{8}\right), \\
& -\tau_{6}=-\left(\alpha_{1}+\alpha_{2}+\alpha_{3}+2 \alpha_{4}+2 \alpha_{5}+2 \alpha_{6}+2 \alpha_{7}+\alpha_{8}\right), \\
& \tau_{7}=\alpha_{1}+2 \alpha_{2}+2 \alpha_{3}+3 \alpha_{4}+2 \alpha_{5}+2 \alpha_{6}+\alpha_{7}+\alpha_{8}, \\
& \tau_{8}=\alpha_{1}+\alpha_{2}+2 \alpha_{3}+3 \alpha_{4}+3 \alpha_{5}+2 \alpha_{6}+\alpha_{7}+\alpha_{8}, \\
& \tau_{9}=\alpha_{1}+\alpha_{2}+2 \alpha_{3}+3 \alpha_{4}+2 \alpha_{5}+2 \alpha_{6}+2 \alpha_{7}+\alpha_{8}, \\
& -\tau_{10}=-\left(2 \alpha_{1}+3 \alpha_{2}+4 \alpha_{3}+6 \alpha_{4}+4 \alpha_{5}+3 \alpha_{6}+2 \alpha_{7}+\alpha_{8}\right), \\
& \tau_{11}=2 \alpha_{1}+3 \alpha_{2}+4 \alpha_{3}+6 \alpha_{4}+5 \alpha_{5}+4 \alpha_{6}+2 \alpha_{7}+\alpha_{8} .
\end{aligned}
$$

It is easy to check the following facts:

(15) The eleven elements $e_{\alpha_{2}}, e_{\tau_{1}}, e_{\alpha_{5}}, e_{-\tau_{2}}, e_{-\tau_{4}}, e_{\tau_{7}}, e_{\alpha_{6}}, e_{-\tau_{3}}, e_{\tau_{11}}$, $e_{\tau_{10}}, e_{\alpha_{8}}$ span a $C_{G}(s)$-submodule $M_{2}$ of $\mathcal{N}_{q, s}$,

(16) The element $e_{\alpha_{1}}$ spans a $C_{G}(s)$-submodule $M_{1}$ of $\mathcal{N}_{q, s}$,

(17) The elements $e_{\alpha_{3}}, e_{-\tau_{6}}$ span a $C_{G}(s)$-submodule $M_{3}$ of $\mathcal{N}_{q, s}$.

(18) The elements $e_{\alpha_{4}}, e_{-\tau_{5}}, e_{\tau_{9}}, e_{\alpha_{7}}$ span a $C_{G}(s)$-submodule $M_{4}$ of $\mathcal{N}_{q, s}$.

Clearly $\mathcal{N}_{q, s}$ is the direct sum of all $M_{i}$. But it is not easy to see the number of $C_{G}(s)$-orbits of $\mathcal{N}_{q, s}$ in this case.

If $o(q)=16$, then $C_{G}(s)$ is generated by $T, U_{ \pm \xi_{i}}, i=1,2,3,4$, here

$$
\begin{aligned}
& \xi_{1}=\alpha_{1}+2 \alpha_{2}+3 \alpha_{3}+4 \alpha_{4}+3 \alpha_{5}+2 \alpha_{6}+\alpha_{7}, \\
& \xi_{2}=\alpha_{1}+\alpha_{2}+2 \alpha_{3}+3 \alpha_{4}+3 \alpha_{5}+3 \alpha_{6}+2 \alpha_{7}+\alpha_{8}, \\
& \xi_{3}=\alpha_{1}+2 \alpha_{2}+2 \alpha_{3}+4 \alpha_{4}+3 \alpha_{5}+2 \alpha_{6}+\alpha_{7}+\alpha_{8}, \\
& \xi_{4}=\alpha_{1}+2 \alpha_{2}+2 \alpha_{3}+3 \alpha_{4}+3 \alpha_{5}+2 \alpha_{6}+2 \alpha_{7}+\alpha_{8} .
\end{aligned}
$$


If $\alpha$ is not simple root, then $\alpha(s)=q$ if and only if $\alpha$ is one of the following roots:

$$
\begin{aligned}
& \eta_{1}=2 \alpha_{1}+2 \alpha_{2}+3 \alpha_{3}+4 \alpha_{4}+3 \alpha_{5}+2 \alpha_{6}+\alpha_{7} \\
& -\eta_{2}=-\left(\alpha_{1}+2 \alpha_{2}+2 \alpha_{3}+4 \alpha_{4}+3 \alpha_{5}+2 \alpha_{6}+\alpha_{7}\right), \\
& -\eta_{3}=-\left(\alpha_{1}+2 \alpha_{2}+2 \alpha_{3}+3 \alpha_{4}+3 \alpha_{5}+2 \alpha_{6}+\alpha_{7}+\alpha_{8}\right), \\
& -\eta_{4}=-\left(\alpha_{1}+2 \alpha_{2}+2 \alpha_{3}+3 \alpha_{4}+2 \alpha_{5}+2 \alpha_{6}+2 \alpha_{7}+\alpha_{8}\right), \\
& -\eta_{5}=-\left(\alpha_{1}+\alpha_{2}+2 \alpha_{3}+3 \alpha_{4}+3 \alpha_{5}+2 \alpha_{6}+2 \alpha_{7}+\alpha_{8}\right), \\
& \eta_{6}=\alpha_{1}+2 \alpha_{2}+2 \alpha_{3}+3 \alpha_{4}+3 \alpha_{5}+3 \alpha_{6}+2 \alpha_{7}+\alpha_{8}, \\
& \eta_{7}=\alpha_{1}+2 \alpha_{2}+2 \alpha_{3}+4 \alpha_{4}+3 \alpha_{5}+2 \alpha_{6}+2 \alpha_{7}+\alpha_{8}, \\
& \eta_{8}=\alpha_{1}+2 \alpha_{2}+3 \alpha_{3}+4 \alpha_{4}+3 \alpha_{5}+2 \alpha_{6}+\alpha_{7}+\alpha_{8} .
\end{aligned}
$$

It is easy to check the following facts:

(19) The elements $e_{\alpha_{1}}, e_{\eta_{1}}$ span a $C_{G}(s)$-submodule $M_{1}$ of $\mathcal{N}_{q, s}$,

(20) The elements $e_{\alpha_{2}}, e_{\alpha_{6}}, e_{\eta_{6}}, e_{-\eta_{5}}$ span a $C_{G}(s)$-submodule $M_{2}$ of $\mathcal{N}_{q, s}$,

(21) The elements $e_{\alpha_{3}}, e_{\alpha_{8}}, e_{\eta_{8}}, e_{-\eta_{2}}$ span a $C_{G}(s)$-submodule $M_{3}$ of $\mathcal{N}_{q, s}$,

(22) The elements $e_{\alpha_{4}}, e_{\alpha_{7}}, e_{\eta_{7}}, e_{-\eta_{3}}$ span a $C_{G}(s)$-submodule $M_{4}$ of $\mathcal{N}_{q, s}$,

(23) The elements $e_{\alpha_{5}}, e_{-\eta_{4}}$ span a $C_{G}(s)$-submodule $M_{5}$ of $\mathcal{N}_{q, s}$,

Clearly $\mathcal{N}_{q, s}$ is the direct sum of all $M_{i}$. Note that $U_{ \pm \xi_{i}}$ act on $M_{1}+M_{3}$ trivially for $i=2,4$ and $U_{ \pm \xi_{3}}$ also act on $M_{1}$ trivially. By direct computation we see that the $C_{G}(s)$-submodule $M_{1}+M_{3}$ has eight $C_{G}(s)$-orbits, representatives of the orbits can be chosen as follows: 0 , $e_{\alpha_{1}}, e_{\alpha_{3}}, e_{\alpha_{3}}+e_{\alpha_{8}}, e_{\alpha_{1}}+e_{\alpha_{3}}, e_{\alpha_{1}}+e_{\alpha_{3}}+e_{\alpha_{8}}, e_{\alpha_{1}}+e_{-\eta_{2}}, e_{\alpha_{1}}+e_{\alpha_{3}}+e_{-\eta_{2}}$. Similarly, one can see that $M_{4}+M_{5}$ has also eight $C_{G}(s)$-orbits, and $M_{2}$ has three $C_{G}(s)$-orbits. Thus the number of $C_{G}(s)$-orbits in $\mathcal{N}_{q, s}$ is not less than $8 \times 8 \times 3=192$.

If $o(q)=17$, then $C_{G}(s)$ is generated by $T, U_{ \pm \eta_{i}}, i=1,6,7,8$, here

$$
\begin{aligned}
& \eta_{1}=2 \alpha_{1}+2 \alpha_{2}+3 \alpha_{3}+4 \alpha_{4}+3 \alpha_{5}+2 \alpha_{6}+\alpha_{7} \\
& \eta_{6}=\alpha_{1}+2 \alpha_{2}+2 \alpha_{3}+3 \alpha_{4}+3 \alpha_{5}+3 \alpha_{6}+2 \alpha_{7}+\alpha_{8}, \\
& \eta_{7}=\alpha_{1}+2 \alpha_{2}+2 \alpha_{3}+4 \alpha_{4}+3 \alpha_{5}+2 \alpha_{6}+2 \alpha_{7}+\alpha_{8}, \\
& \eta_{8}=\alpha_{1}+2 \alpha_{2}+3 \alpha_{3}+4 \alpha_{4}+3 \alpha_{5}+2 \alpha_{6}+\alpha_{7}+\alpha_{8} .
\end{aligned}
$$

If $\alpha$ is not simple root, then $\alpha(s)=q$ if and only if $\alpha$ is one of the following roots: 


$$
\begin{aligned}
& -\xi_{1}=-\left(\alpha_{1}+2 \alpha_{2}+3 \alpha_{3}+4 \alpha_{4}+3 \alpha_{5}+2 \alpha_{6}+\alpha_{7}\right), \\
& -\xi_{2}=-\left(\alpha_{1}+\alpha_{2}+2 \alpha_{3}+3 \alpha_{4}+3 \alpha_{5}+3 \alpha_{6}+2 \alpha_{7}+\alpha_{8}\right), \\
& -\xi_{3}=-\left(\alpha_{1}+2 \alpha_{2}+2 \alpha_{3}+4 \alpha_{4}+3 \alpha_{5}+2 \alpha_{6}+\alpha_{7}+\alpha_{8}\right), \\
& -\xi_{4}=-\left(\alpha_{1}+2 \alpha_{2}+2 \alpha_{3}+3 \alpha_{4}+3 \alpha_{5}+2 \alpha_{6}+2 \alpha_{7}+\alpha_{8}\right), \\
& \xi_{5}=2 \alpha_{1}+2 \alpha_{2}+3 \alpha_{3}+4 \alpha_{4}+3 \alpha_{5}+2 \alpha_{6}+\alpha_{7}+\alpha_{8}, \\
& \xi_{6}=\alpha_{1}+2 \alpha_{2}+3 \alpha_{3}+4 \alpha_{4}+3 \alpha_{5}+2 \alpha_{6}+2 \alpha_{7}+\alpha_{8}, \\
& \xi_{7}=\alpha_{1}+2 \alpha_{2}+2 \alpha_{3}+4 \alpha_{4}+3 \alpha_{5}+3 \alpha_{6}+2 \alpha_{7}+\alpha_{8} .
\end{aligned}
$$

It is easy to check the following facts:

(24) The elements $e_{\alpha_{2}}, e_{-\xi_{2}}$ span a $C_{G}(s)$-submodule $M_{2}$ of $\mathcal{N}_{q, s}$,

(25) The element $e_{\alpha_{5}}$ spans a $C_{G}(s)$-submodule $M_{5}$ of $\mathcal{N}_{q, s}$,

(26) The elements $e_{\alpha_{1}}, e_{-\xi_{1}}, e_{\xi_{5}}, e_{\alpha_{8}}$ span a $C_{G}(s)$-submodule $M_{1}$ of $\mathcal{N}_{q, s}$,

(23) The elements $e_{\alpha_{3}}, e_{-\xi_{3}}, e_{\xi_{6}}, e_{\alpha_{7}}$ span a $C_{G}(s)$-submodule $M_{3}$ of $\mathcal{N}_{q, s}$,

(24) The elements $e_{\alpha_{4}}, e_{-\xi_{4}}, e_{\xi_{7}}, e_{\alpha_{6}}$ span a $C_{G}(s)$-submodule $M_{4}$ of $\mathcal{N}_{q, s}$.

Clearly $\mathcal{N}_{q, s}$ is the direct sum of all $M_{i}$. Noting that $M_{i}$ has three $C_{G}(s)$-orbits for $i=4,5, M_{5}$ has two $C_{G}(s)$-orbits, $M_{2}+M_{4}$ has eight $C_{G}(s)$-orbits, we see that the number of $C_{G}(s)$-orbits in $\mathcal{N}_{q, s}$ is not less than $3 \times 3 \times 8 \times 2=144$.

Completely similar to the above discussions, one see that the $C_{G}(s)$ orbits in $\mathcal{N}_{q, s}$ is not less than 144 if $o(q)=19,21,22,23,25,26,27,28,29$.

\section{Proof of Theorem 1.2.}

In this section we prove Theorem 1.2, the main result of this article.

First assume that $\sum_{w \in W_{0}} q^{l(w)} \neq 0$.

Assume that $\psi: H_{q} \rightarrow \mathbb{C}[W]=H_{1}$ is an isomorphism of $\mathbb{C}$ algebras. Then $\phi$ induces an isomorphism from $Z\left(H_{q}\right) \rightarrow Z\left(H_{1}\right)$. Let $s$ be a semisimple element in $T$. Composing the homomorphism $\phi_{1, s}$ : $Z(\mathbb{C}[W]) \rightarrow \mathbb{C}$ with $\psi$, we get a homomorphism $\phi_{1, s} \psi: Z\left(H_{q}\right) \rightarrow \mathbb{C}$. So there exists a semisimple element $t$ in $T$ such that $\phi_{1, s} \psi=\phi_{q, t}$. This induces an isomorphism $H_{q, t} \rightarrow H_{1, s}$.

Assume further that $H_{1, s}$ has one dimensional representation, then $H_{q, t}$ has also one dimensional representation. So the number of the 
central characters $\phi_{q, t}(t \in T)$ which admit one-dimensional representations is equal to the number of the central characters $\phi_{1, s}(s \in T)$ which admit one-dimensional representations. According to Corollary 2.2 and Corollary 3.3, this is not true if $R$ is not simply laced. So the theorem is true if $\sum_{w \in W_{0}} q^{l(w)} \neq 0$ and $R$ is not simply laced.

Now assume that $R$ is simply laced. Let $s, t$ be as above. By 2.3 (a), the number $\left|\operatorname{Irr} H_{1, s}\right|$ of isomorphism classes of irreducible representations of $H_{1, s}$ is equal to the number $\left|\operatorname{Irr} W_{0}\right|$ of isomorphism classes of irreducible complex representations of the Weyl group $W_{0}$. We will show that $\left|\operatorname{Irr} W_{0}\right|$ is not equal to the number $\left|\operatorname{Irr} H_{q, t}\right|$ of isomorphism classes of irreducible representations of $H_{q, t}$. We do this case by case.

Type $A_{n}(n \geq 2)$. It is known that $\left|\operatorname{Irr} W_{0}\right|$ is the number $p(n+1)$ of partitions of $n+1$. Since $\sum_{w \in W_{0}} q^{l(w)} \neq 0$, the order of $q$ is greater than $n$. By Lemma 3.4, we see that $\left|\operatorname{Irr} H_{q, t}\right|=2^{n}$. It suffices to show that $\left|\operatorname{Irr} H_{q, t}\right|=2^{n}>p(n+1)=\left|\operatorname{Irr} W_{0}\right|$ if $n \geq 2$.

One can use Euler's Pentagonal Theorem to prove $2^{n}>p(n+1)$ for $n \geq 2$. Set $p(0)=1$ and $p(m)=0$ if $m<0$. The theorem says

$$
p(n)=\sum_{k=1}^{\infty}(-1)^{k+1}\left(p\left(n-\frac{k(3 k-1)}{2}\right)+\left(p\left(n-\frac{k(3 k+1)}{2}\right)\right),\right.
$$

which implies that $p(n) \leq p(n-1)+p(n-2)$ for $n \geq 2$. One can also use induction on $n$ to prove the result directly. When $n=2,3,4,5, p(n+1)$ is $3,5,7,11$ respectively. The result is true in these cases. Now assume that $k \geq 5$ and the result is true for integer $n$ satisfying $2 \leq n \leq k$. For $1 \leq i \leq k+2$, let $P_{i}$ be the number of partitions $a_{1} \geq a_{2} \geq \cdots \geq a_{m}$ of $k+2$ such that $a_{1}=i$. Then $P_{k+2}=P_{k+1}=P_{1}=1, P_{k}=2, P_{k-1}=3$. By induction hypothesis, $2^{k+1-i}>P_{i}$ whenever $2 \leq i \leq k-2$. Hence,

$$
\begin{aligned}
p(k+2) & =P_{k+2}+P_{k+1}+P_{k}+P_{k-1}+\cdots+P_{2}+P_{1} \\
& =8+P_{k-2}+P_{k-3}+\cdots+P_{3} \\
& <8+2^{3}+2^{4}+\cdots+2^{k-1} \\
& =2^{k}<2^{k+1} .
\end{aligned}
$$

Type $D_{n}(n \geq 4)$. It is known that $\left|\operatorname{Irr} W_{0}\right|$ is the number $\mathcal{P}(n)$ of unordered pairs $(\xi, \eta)$ of partitions $\xi, \eta$ with $|\xi|+|\eta|=n$, and any pairs $(\xi, \eta)$ with $\xi=\eta$ and $|\xi|+|\eta|=n$ is counted twice, see 2.3. By 1.6(b) and the discussion in 3.5, we have $\left|\operatorname{Irr} H_{q, t}\right| \geq 2^{4} \cdot 3^{\frac{n-4}{2}}$ if $n \geq 4$ is even 
and $\left|\operatorname{Irr} H_{q, t}\right| \geq 2^{5} \cdot 3^{\frac{n-5}{2}}$ if $n \geq 5$ is odd. Define $\mathcal{D}(n)(n \geq 4)$ to be $2^{4} \cdot 3^{\frac{n-4}{2}}$ if $n$ is even and to be $2^{5} \cdot 3^{\frac{n-5}{2}}$ if $n$ is odd. It suffices to show that $\mathcal{D}(n)>\mathcal{P}(n)$ if $n \geq 4$.

We first show that (*) $p(n) \leq 2 p(n-2)$ if $n \geq 8$.

Let $Q_{n}$ be the set of all partitions of $n$. For $1 \leq k \leq n$, let $Q_{n, k}$ be the set of partitions of $n$ with smallest term $k$ and $p(n, k)$ be the cardinality of $Q_{n, k}$. Then $p(n, 1)=p(n-1)$ and $p(n)$ is the sum of all $p(n, k)$. So $p(n)-p(n-1)$ is the sum of all $p(n, k)$ with $k \geq 2$. Since $p(n)-p(n-2)=p(n)-p(n-1)+p(n-1)-p(n-2)$, we get

$$
p(n)-p(n-2)=\sum_{k=2}^{n} p(n, k)+\sum_{k=2}^{n-1} p(n-1, k) .
$$

We define an injection

$$
\tau:\left(\bigcup_{k=2}^{n} Q_{n, k}\right) \cup\left(\bigcup_{k=2}^{n-1} Q_{n-1, k}\right) \rightarrow \bigcup_{k=1}^{n-2} Q_{n-2, k}=Q_{n} .
$$

For a partition $a_{1} \geq a_{2} \geq a_{3} \cdots \geq a_{r}>0$ of $n$, we shall simply write it as $a_{1} a_{2} a_{3} \cdots a_{r}$. For $A=a_{1} a_{2} \cdots a_{r-1} a_{r} \in Q_{n-1, k}(k \geq 2)$, define $\tau(A)=a_{1} a_{2} \cdots a_{r-1}\left(a_{r}-1\right)$. Note that $a_{r-1}>a_{r}-1 \geq 1$.

For $A=a_{1} a_{2} \cdots a_{r-1} a_{r} \in Q_{n, k}(k \geq 4)$, define $\tau(A)=a_{1} a_{2} \cdots a_{r-1} 1 \cdots 1$, where 1 appears $a_{r}-2=k-2$ times. Note that $a_{r-1}-\left(a_{r}-2\right) \geq 2$.

For $A=a_{1} a_{2} \cdots a_{r-2} a_{r-1} 3 \in Q_{n, 3}$, set $\tau(A)=a_{1} a_{2} \cdots a_{r-2} 21 \cdots 1$, where 1 appears $a_{r-1}-1$ times. Note that $a_{r-1}-1 \geq 2$ and $a_{r-2} \geq 3$.

For $r \geq 3$ and $A=a_{1} a_{2} \cdots a_{r-3} a_{r-2} a_{r-1} 2 \in Q_{n, 2}$, set $\tau(A)=$ $a_{1} a_{2} \cdots a_{r-3} a_{r-2} a_{r-1}$ if $a_{r-2}=a_{r-1}$ and set $\tau(A)=a_{1} a_{2} \cdots a_{r-3}\left(a_{r-1}+\right.$ 1) $1 \cdots 1$ if $a_{r-2}>a_{r-1}$, where 1 appears $a_{r-2}-1$ times. Note that if $a_{r-2}>a_{r-1}$, then $a_{r-2}-1 \geq 2$ and $a_{r-1}+1-\left(a_{r-2}-1\right) \leq a_{r-2}-$ $\left(a_{r-2}-1\right) \leq 1$.

For $A=a_{1} 2 \in Q_{n, 2}$, let $\tau(A)=\left(a_{1}-6\right) 2211$. Since $n \geq 8, \tau(A)$ is well defined.

It is easy to check that $\tau$ is injective. Hence we have $p(n)-p(n-2) \leq$ $p(n-2)$ if $n \geq 8$.

Now we show that $\mathcal{D}(n)>\mathcal{P}(n)$. We use induction on $n$. When $n=4,5,6,7,8,9,10,11,12, \mathcal{P}(n)$ is $13,18,37,55,100,150,251,376$, 
599 , respectively, and $\mathcal{D}(n)$ is $16,32,48,96,144,288,432,864,1296$, respectively. So the result is true in these cases.

Since $\mathcal{D}(n+2)=3 \mathcal{D}(n)$, it suffices to show that $\mathcal{P}_{n+2}<3 \mathcal{P}(n)$ for $n \geq 11$.

Assume that $n=2 k \geq 12$. Then

$$
\begin{gathered}
\mathcal{P}(n)=\sum_{i=0}^{k-1} p(n-i) p(i)+\frac{p(k)(p(k)+3)}{2}, \\
\mathcal{P}(n+2)=\sum_{i=0}^{k} p(n+2-i) p(i)+\frac{p(k+1)(p(k+1)+3)}{2} .
\end{gathered}
$$

It is well known that $p(k+1) \leq \frac{1}{2}(p(k+2)+p(k))$. Since $k+2 \geq 8$, by $(*)$, we get $p(k+1) \leq \frac{3}{2} p(k)$. Again by $(*)$, for $0 \leq i \leq k-1$, we have $p(n+2-i) \leq 2 p(n-i)$ since $n+2-i=2 k+2-i>k+3>8$. Thus $3 \mathcal{P}(n)-\mathcal{P}(n+2) \geq \sum_{i=0}^{k-1} p(n-i) p(i)+\frac{3}{8} p(k)^{2}+\frac{9}{4} p(k)-p(k+2) p(k)$.

Euler's Pentagonal Theorem implies that $p(k) \leq p(k-1)+p(k-2)$. Hence $3 \mathcal{P}(n)-\mathcal{P}(n+2) \geq \sum_{i=0}^{k-3} p(n-i) p(i)+p(k+1) p(k-1)+\frac{3}{8} p(k)^{2}+\frac{9}{4} p(k)-p(k+2) p(k-1)$.

But $p(k+2) \leq \frac{3}{2} p(k+1)$, so $3 \mathcal{P}(n)-\mathcal{P}(n+2) \geq \sum_{i=0}^{k-3} p(n-i) p(i)+\frac{3}{8} p(k)^{2}+\frac{9}{4} p(k)-\frac{1}{2} p(k+1) p(k-1)$.

When $k-1 \geq 8$, we have $p(k-1) \leq 2 p(k-3)$, so $p(k+3) p(k-3)>$ $\frac{1}{2} p(k+1) p(k-1)$ if $k \geq 9$. If $6 \leq k<9$, one may check directly that $p(k+3) p(k-3)>\frac{1}{2} p(k+1) p(k-1)$. So $3 \mathcal{P}(n)>3 \mathcal{P}(n+2)$ if $n=2 k \geq 12$.

If $n=2 k+1 \geq 11$, the argument for $3 \mathcal{P}(n)>\mathcal{P}(n+2)$ is similar (and simpler). The proof for type $D_{n}$ is complete.

Type $E_{6}, E_{7}, E_{8}$. The number $\left|\operatorname{Irr} W_{0}\right|$ is $25,60,112$, respectively, see 2.3. Using 1.5 (b), 2.3, Lemma 3.4 and the discussion in 3.5, we see that $\left|\operatorname{Irr} H_{q, t}\right|>\left|\operatorname{Irr} W_{0}\right|$ if $o(q) \neq 11,13$ when $R$ is type $E_{8}$. 
In the rest part of the proof we assume that $\sum_{w \in W_{0}} q^{l(w)}=0$. Then any simple quotient module of certain standard modules of $H_{q}$ is a simple constituent of some other standard modules, see [X1, Theorem 7.8, p.83]. This indicates that the standard modules of $H_{q}$ and the standard modules of $H_{1}$ behave differently, so $H_{q}$ and $H_{1}$ should not be isomorphic. We make this point explicit.

Let $t \in T$ be such that $H_{q, t}$ has a one-dimensional representation on which all $T_{r}$ act by scalar $q$. Assume that $H_{q}$ and $H_{1}$ were isomorphic. Then there exists $s \in T$ such that $H_{q, t}$ and $H_{1, s}$ are isomorphic. By Lemma 3.1, we may assume that $\alpha(t)=q$ for all simple roots $\alpha$. By Lemma 2.1, $\alpha(s)=1$ for all simple roots $\alpha$.

Let $D=\sum_{w \in W_{0}} T_{w} \in H_{q}$ and $D^{\prime}=\sum_{w \in W_{0}}(-q)^{-l(w)} T_{w} \in H_{q}$. We also use $D$ and $D^{\prime}$ for their images in $H_{q, t}$ respectively. Let $C=$ $\sum_{w \in W_{0}} w \in H_{1}=\mathbb{C}[W]$ and $C^{\prime}=\sum_{w \in W_{0}}(-1)^{-l(w)} w \in H_{1}$. The images in $H_{1, s}$ of $C$ and $C^{\prime}$ will again be denoted by $C$ and $C^{\prime}$ respectively.

According to [X3, Theorem 3.3], $\mathrm{CH}_{1, s} \mathrm{C}^{\prime}$ is a one-dimensional twosided ideal of $H_{1, s}$. Let $\pi: H_{1, s} \rightarrow H_{q, t}$ be an isomorphism. Then $\pi\left(C H_{1, s} C^{\prime}\right)=\pi(C) H_{q, t} \pi\left(C^{\prime}\right)$ is a one-dimensional two-sided ideal of $H_{q, t}$. Let $v$ be a nonzero element of $\pi\left(\mathrm{CH}_{1, s} \mathrm{C}^{\prime}\right)$. Then one of the following cases must happen:

(1) $T_{w} v=v T_{w}=q^{l(w)} v$ for all $w \in W_{0}$,

(2) $T_{w} v=v T_{w}=(-1)^{l(w)} v$ for all $w \in W_{0}$,

(3) $T_{w} v=q^{l(w)} v, v T_{w}=(-1)^{l(w)} v$ for all $w \in W_{0}$,

(4) $T_{w} v=(-1)^{l(w)} v, v T_{w}=q^{l(w)} v$ for all $w \in W_{0}$.

To go further we need the following facts.

(5) Let $h \in H_{q}$. If $T_{r} h=-h\left(\right.$ resp. $\left.h T_{r}=-h ; T_{r} h=q h ; h T_{r}=q h\right)$ for all simple reflection $r$ in $W_{0}$, the $h \in D^{\prime} H_{q}$ (resp. $h \in H_{q} D^{\prime}$; $\left.h \in H_{q} D ; h \in D H_{q}\right)$.

We explain the reasons for (5). For $w \in W$, let $D_{w}^{\prime}=\sum_{y \leq w}(-q)^{-l(y)} P_{y, w}\left(q^{-1}\right) T_{y}$, where $\leq$ stands for the Bruhat order on $W$, and $P_{y, w}$ are the KazhdanLusztig polynomials. Then the elements $D_{w}^{\prime}, w \in W_{0}$, form a basis of the subalgebra $H_{q, W_{0}}$ of $H_{q}$ generated by all $T_{w}, w \in W_{0}$. Let $w \in W_{0}$ and $r$ be a simple reflection in $W_{0}$. Then $T_{r} D_{w}^{\prime}=-D_{w}^{\prime}$ (resp. $D_{w}^{\prime} T_{r}=-D_{w}^{\prime}$ ) if and only if $r w \leq w$ (resp. $\left.w r \leq w\right)$. Moreover, 
if $r w \geq w$, then $T_{r} D_{w}^{\prime}=q^{\frac{3}{2}} D_{r w}^{\prime}+\sum_{y \leq w} a_{y} D_{y}^{\prime}, a_{y} \in \mathbb{C}$. See (2.3.a) and (2.3.c) in [KL1]. Note that the elements $D_{w}^{\prime} \theta_{x}, w \in W_{0}, x \in X$, form a basis of $H_{q}$. Using these facts we see easily that $h \in D^{\prime} H_{q}$ if $h \in H_{q}$ and $T_{r} h=-h$ for all simple reflections $r$ in $W_{0}$. The arguments for other parts of (5) are similar.

The following statement is easy to check.

(6) Let $r$ be a simple reflection in $W_{0}$ and $\theta=\sum_{x \in X} a_{x} \theta_{x} \in \Theta_{q}$, where $a_{x} \in \mathbb{C}$. If $D \theta T_{r}=q D \theta$ (resp. $D^{\prime} \theta T_{r}=-D^{\prime} \theta$ ), then $a_{x}=a_{r(x)}$ and $T_{r} \theta=\theta T_{r}$.

As a consequence of (5) and (6), we get (see also [L1, p.213], and note also that $D H_{q} D \subset D Z\left(H_{q}\right)$ by [L1, Proposition 8.6])

(7) Let $h \in H_{q}$. If $T_{r} h=h T_{r}=q h\left(\right.$ resp. $\left.T_{r} h=h T_{r}=-h\right)$ for all simple reflections $r$ in $W_{0}$, then $h$ is in $D Z\left(H_{q}\right)$ (resp. $\left.D^{\prime} Z\left(H_{q}\right)\right)$.

The following result is implicit in [L1, p.213, line -7]).

(8) Assume that $q \neq-1$. Let $h \in H_{q}$. If $T_{r} h=-h$ and $h T_{r}=q h$ for all simple reflections $r$ in $W_{0}$, then $h$ is in $D^{\prime} H_{q} D$.

Now we argue for (8). Assume that $h \neq 0$. By (5), $h$ is in $H_{q} D$. Let $\Omega=\left\{w \in W \mid w r \geq w\right.$ for all simple reflections $r$ in $\left.W_{0}\right\}$. According to [X3, Lemma 2.2], the elements $D_{w}^{\prime} D, w \in \Omega$, form a basis of $H_{q} D$. Let $h=\sum_{w \in \Omega} \xi_{w} D_{w}^{\prime} D, \xi_{w} \in \mathbb{C}$. Assume that $r$ is a simple reflection in $W_{0}$. Write $\left(T_{r}-q\right) h=\sum_{w \in \Omega} \eta_{w} D_{w}^{\prime} D, \eta_{w} \in \mathbb{C}$. Note that for any $w \in W$ we have $\left(T_{r}-q\right) D_{w}^{\prime}=-(1+q) D_{w}^{\prime}$ if $r w \leq w$ and $\left(T_{r}-q\right) D_{w}^{\prime}=$ $q^{\frac{3}{2}} D_{r w}^{\prime}+\sum_{y<w} a_{y} D_{y}^{\prime}, a_{y} \in \mathbb{C}$ if $r w \geq w$. Moreover, $r y \leq y$ if $a_{y} \neq 0$ (see $[\mathrm{KL} 1,(2.3 . \mathrm{a})]$. Since $D_{y}^{\prime} D=0$ for any $y \notin \Omega$, we must have $\eta_{w}=0$ if $r w \geq w$. But $\left(T_{r}-q\right) h=-(1+q) h \neq 0$, so we must have $r w \leq w$ if $\xi_{w} \neq 0$. Therefore, $r w \leq w$ for all simple reflections $r$ in $W_{0}$ whenever $\xi_{w} \neq 0$. By $(5)$, then $D_{w}^{\prime} \in D^{\prime} H_{q}$ if $\xi_{w} \neq 0$. So $h$ is $D^{\prime} H_{q} D$.

There is a unique involutive automorphism $\xi \rightarrow \xi^{*}$ of the $\mathbb{C}$-algebra $H_{q}$ such that $T_{r}^{*}=-q T_{r}^{-1}=q-1-T_{r}\left(r \in S \cap W_{0}\right), \theta_{x}^{*}=\theta_{x^{-1}}(x \in$ $X)[\mathrm{KL} 2,2.13(\mathrm{~d})]$. Noting that $D^{*}=(-1)^{l\left(w_{0}\right)} D^{\prime}$, we see that $(8)$ is equivalent to the following result.

(9) Let $h \in H_{q}$. If $T_{r} h=q h$ and $h T_{r}=-h$ for all simple reflections $r$ in $W_{0}$, then $h$ is in $D H_{q} D^{\prime}$. 
By (7), (8) and (9), we must have $v \in \mathbb{C} D$, or $v \in \mathbb{C} D^{\prime}$, or $v \in$ $D H_{q, t} D^{\prime}$, or $v \in D^{\prime} H_{q, t} D$. But neither $\mathbb{C} D$ nor $\mathbb{C} D^{\prime}$ is two-sided ideal of $H_{q, t}$. So cases (1) and (2) would not occur. Since $\sum_{w \in W_{0}} q^{l(w)}=0$, by $\left[\mathrm{X} 3\right.$, Theorem 3.3], $D H_{q, t} D^{\prime}=D^{\prime} H_{q, t} D=0$. These contradict that $v \neq 0$. So $H_{q, t}$ and $H_{1, s}$ are not isomorphic. Therefore, $H_{q}$ and $H_{1}$ are not isomorphic.

The theorem is proved.

Acknowledgement: Part of the work was done during the visit of $\mathrm{N}$. Xi to the Department of Mathematics, Nagoya University, Xi thanks the department for hospitality and financial support. We are very grateful to the referee for carefully reading and valuable comments, which lead significant improvement of the paper.

\section{References}

[G] A. Gyoja, Modular representation theory over a ring of higher dimension with applications to Hecke algebras. J. Algebra 174 (1995), no. 2, 553-572.

[I] N. Iwahori,On the structure of a Hecke ring of a Chevalley group over a finite field. J. Fac. Sci. Univ. Tokyo Sect. I 101964 (1964) 215-236.

[IM] N. Iwahori and H. Matsumoto, On some Bruhat decomposition and the structure of Hecke rings of p-adic Chevalley groups. Publ. IHES 25 (1965), $5-48$.

[K] Shin-ichi Kato, A realization of irreducible representations of affine Weyl groups. Nederl. Akad. Wetensch. Indag. Math. 45 (1983), no. 2, 193-201.

[KL1] D. Kazhdan and G. Lusztig, Representations of Coxeter groups and Hecke algebras. Invent. Math. 53 (1979), 165-184.

[KL2] D. Kazhdan and G. Lusztig, Proof of the Deligne-Langlands conjecture for Hecke algebras. Invent. Math. 87 (1987), no. 1, 153-215.

[L1] G. Lusztig, Singularities, character formulas, and a q-analog of weight multiplicities. Astérisque 101-102 (1983), pp.208-227.

[L2] G. Lusztig, Some examples of square integrable representations of semisimple pp-adic groups. Trans. Amer. Math. Soc. 277 (1983), no. 2, 623-653.

[L3] G. Lusztig, Representations of affine Hecke algebras. in "Orbites unipotentes et representations, II". Astérisque No. 171-172 (1989), 73-84.

[S] R. Steinberg, On a theorem of Pittie. Topology 14 (1975), 173-177.

[X1] N. Xi, Representations of affine Hecke algebras. Lecture Notes in Mathematics 1587, Springer-Verlag, 1994.

[X2] N. Xi, Representations of affine Hecke algebras and based rings of affine Weyl groups. J. Amer. Math. Soc. 20 (2007), 211-217.

[X3] N. Xi, Canonical left cells and the lowest two-sided cell of an affine Weyl group. Arxiv1106.3654v2.

[Y] R. Yan, Isomorphisms between affine Hecke algebras of type $\tilde{A}_{2}$. J. Algebra 324 (2010), no. 5, 984-999. 
* Graduate School of Mathematics, Nagoya University, ChikusaKU, NAGOYA, 464-8602 JAPAN

E-mail address: shoji@math.nagoya-u.ac.jp

$\dagger$ Institute of Mathematics, Chinese Academy of Sciences, Beijing, 100190, CHINA

E-mail address: nanhua@math.ac.cn 